\title{
GROUPOIDS AND AN INDEX THEOREM FOR CONICAL PSEUDO-MANIFOLDS
}

\author{
CLAIRE DEBORD, JEAN-MARIE LESCURE, AND VICTOR NISTOR
}

\begin{abstract}
We define an analytical index map and a topological index map for conical pseudomanifolds. These constructions generalize the analogous constructions used by Atiyah and Singer in the proof of their topological index theorem for a smooth, compact manifold $M$. A main ingredient is a noncommutative algebra that plays in our setting the role of $C_{0}\left(T^{*} M\right)$. We prove a Thom isomorphism between non-commutative algebras which gives a new example of wrong way functoriality in $K$-theory. We then give a new proof of the Atiyah-Singer index theorem using deformation groupoids and show how it generalizes to conical pseudomanifolds. We thus prove a topological index theorem for conical pseudomanifolds.
\end{abstract}

\section{Contents}

\begin{tabular}{|lr|}
\hline Introduction & 1 \\
\hline 1. Cones and stratified bundles & 4 \\
\hline 2. Lie groupoids and their Lie algebroids & 6 \\
\hline 3. A non-commutative tangent space for conical pseudomanifolds & 10 \\
\hline 4. The analvtical index & 13 \\
\hline 5. The inverse Thom map & 15 \\
\hline 6. Index theorem & 16 \\
\hline References & 25 \\
\hline
\end{tabular}

\section{INTRODUCTION}

Let $V$ be a closed smooth manifold and $P$ an elliptic pseudo-differential operator acting on Sobolev sections of vector bundles over $V$. The ellipticity of $P$ ensures that $P$ has finite dimensional kernel and cokernel. The difference

$$
\text { Ind } P:=\operatorname{dim}(\operatorname{Ker} P)-\operatorname{dim}(\text { Coker } P)
$$

is called the Fredholm index of $P$, and turns out to depend only on the $K$-theory class $[\sigma(P)] \in K^{0}\left(T^{*} V\right)$ of the principal symbol of $P$. We always use $K$-theory with compact supports. Since every element in $K^{0}\left(T^{*} V\right)$ can be represented by the principal symbol of an elliptic pseudo-differential operator, one obtains in this way a group morphism

$$
\begin{array}{ccc}
K^{0}\left(T^{*} V\right) & \longrightarrow & \mathbb{Z} \\
{[\sigma(P)]} & \mapsto & \text { Ind } P
\end{array}
$$

Date: February 11, 2019. 
called the analytical index. This map, defined by M. Atiyah and I. Singer seems to depend essentially on the analysis of elliptic equations, but the main result in [2] is that the index map can be also defined in a purely topological terms in terms of embedding of $V$ in Euclidean spaces. This definition leads to the so called topological index of Atiyah and Singer and the main result of 2 is that the topological index map and the Fredholm index map Ind coincide. See [13] for review of these results, including an extension to non-compact manifolds.

The equality of the topological and Fredholm indices then allowed M. Atiyah and I. Singer to obtain a formula for the index of an elliptic operator $P$ in terms of the Chern classes of $[\sigma(P)]$. Their formula, the celebrated Atiyah-Singer Index Formula, involves, in addition to the Chern character of the principal symbol of $P$, also a universal characteristic class associated with the manifold, the so called Todd class of the given manifold.

It is a natural and important question then to search for extensions of the AtiyahSinger results. It is not the place here to mention all existing generalizations of the Atiyah-Singer index theory, but let us mention here the fundamental work on Connes on foliations [14, 15, 16, 17, 18, as well as 66, 26, 45, 46]. The index theorem for families and Bismut's superconnection formalism play an important role in the study of the so called "anomalies" in physics [7, 8, 9, 24]. A different but related direction is to extend this theory to singular spaces [3. An important step in the index problem on singular manifolds was made by Melrose 36, 37 and Schulze 51, 52 who have introduced the "right class of pseudodifferential operators" for index theory on singular spaces. See also [1, 23, 22, 35, 44, 53,. Generalizations of this theory to singular spaces may turn out to be useful in the development of efficient numerical methods [5].

In this paper, we shall focus on the case of a pseudomanifold $X$ with isolated conical singularities. In earlier work [21, the first two authors defined a $C^{*}$-algebra $A_{X}$ that is dual to the algebra of continuous functions on $X$ from the point of view of $K$-theory (i.e. $A_{X}$ is a " $K$-dual of $X$ " in the sense of [16, 18, 31]), which implies that there exists a natural isomorphism

$$
K_{0}(X) \stackrel{\Sigma_{X}}{\longrightarrow} K_{0}\left(A_{X}\right)
$$

between the $K$-homology of $X$ and the $K$-theory of $T^{S} X$. The $C^{*}$-algebra $A_{X}$ is the $C^{*}$-algebra of a groupoid denoted $T^{S} X$.

One of the main results in [33, see also [38, 43, 42, 50, for similar results using different methods, is that the inverse of the map $\Sigma_{X}$ of Equation (0.1) can be realized, as in the smooth case, by a map that assigns to each element in $K_{0}\left(A_{X}\right)$ an elliptic operator. Thus elements of $K_{0}\left(A_{X}\right)$ can be viewed as the symbols of some natural elliptic pseudodifferential operators realizing the $K$-homology of $X$. Of course, in the singular setting, one has to explain what is meant by "elliptic operator" and by "symbol" on $X$. An example of a convenient choice of elliptic operator in our situation is an elliptic pseudodifferential operator in the $b$-calculus [36. 51] or Melrose's c-calculus. As for the symbols, the notion is more or less the same as in the smooth case. On a manifold $V$, a symbol is a function on $T^{*} V$. For us, it will be convenient to view a symbol as a pointwise multiplication operator on $C_{c}^{\infty}\left(T^{*} V\right)$. A Fourier transform will allow us then to see a symbol as a family of convolution operators on $C_{c}^{\infty}\left(T_{x} V\right), x \in V$. Thus symbols on $V$ appear to be pseudo-differential operators on the groupoid $T V$. This picture generalizes then 
right away to our singular setting. In particular, it leads to a good notion of symbol for conical pseudomanifolds and enables us to interpret 0.1 as the principal symbol map.

In order to better explain our results, we need to introduce some notation. If $G$ is an amenable groupoid, we let $K^{0}(G)$ denote $K_{0}\left(C^{*}(G)\right)$. The analytical index is then defined exactly as in the regular case by

$$
\begin{array}{cccc}
\operatorname{Ind}_{a}^{X}: & K^{0}\left(T^{\varsigma} X\right) & \rightarrow & \mathbb{Z} \\
{[a]} & \mapsto & \operatorname{Ind}\left(\Sigma_{X}^{-1}(a)\right),
\end{array}
$$

where Ind : $K_{0}(X) \rightarrow \mathbb{Z}$ is the usual Fredholm index on compact spaces. Moreover one can generalise the tangent groupoid of A. Connes to our situation and get a nice description of the analytical index.

Following the spirit of [2], we define in this article a topological index $\operatorname{Ind}_{t}^{X}$ that generalizes the classical one and which satisfies the equality:

$$
\operatorname{Ind}_{a}^{X}=\operatorname{Ind}_{t}^{X} .
$$

In fact, we shall see that all ingredients of the classical topological index have a natural generalisation to the singular setting.

- Firstly the embedding of a smooth manifold into $\mathbb{R}^{N}$ gives rise to a normal bundle $N$ and a Thom isomorphism $K^{0}\left(T^{*} V\right) \rightarrow K^{0}\left(T^{*} N\right)$. In the singular setting we embed $X$ into $\mathbb{R}^{N}$, viewed as the cone over $\mathbb{R}^{N-1}$. This gives rise to a conical vector bundle which is a conical pseudomanifold called the normal space and we get an isomorphism: $K^{0}\left(T^{\mathrm{S}} X\right) \rightarrow K^{0}\left(T^{\mathrm{S}} N\right)$. This map restrict to the usual Thom isomorphism on the regular part and is called again the Thom isomorphism.

- Secondly, in the smooth case, the normal bundle $N$ identifies with an open subset of $\mathbb{R}^{N}$, and thus provides an excision map $K(T N) \rightarrow K\left(T \mathbb{R}^{N}\right)$. The same is true in the singular setting : $T^{\mathrm{S}} N$ appears to be an open subgroupoid of $T^{S} \mathbb{R}^{N}$ so we have an excision map $K^{0}\left(T^{\mathrm{S}} N\right) \rightarrow K^{0}\left(T^{\mathrm{S}} \mathbb{R}^{N}\right)$.

- Finally, using the Bott periodicity $K^{0}\left(T^{*} \mathbb{R}^{N}\right) \simeq K^{0}\left(\mathbb{R}^{2 N}\right) \rightarrow \mathbb{Z}$ and a natural $K K$-equivalence between $T^{\mathrm{S}} \mathbb{R}^{N}$ with $T \mathbb{R}^{N}$ we obtain an isomorphism $K^{0}\left(T^{\mathrm{S}} \mathbb{R}^{N}\right) \rightarrow$ $\mathbb{Z}$.

As for the usual definition of the topological index, this allows us to define our generalisation of the topological $\operatorname{Ind}_{t}$ for conical manifolds.

This construction of the topological index is inspired from the techniques of $d e$ formation groupoids introduced by M. Hilsum and G. Skandallis in 27. Moreover, the demonstration of the equality between $\operatorname{Ind}_{a}$ and $\operatorname{Ind}_{t}$ will be the same in the smooth and in the singular setting with the help of deformation groupoids.

We claim that our index maps are straight generalisations of the classical ones. To make this claim more concrete, consider a closed smooth manifold $V$ and choose a point $c \in V$. Take a neighborhood of $c$ diffeomorphic to the unit ball in $\mathbb{R}^{n}$ and consider it as the cone over $S^{n-1}$. This provides $V$ with the structure of a conical manifold. Then the index maps $\operatorname{Ind}_{*}^{\mathrm{S}}: K^{0}\left(T^{\mathrm{S}} V\right) \rightarrow \mathbb{Z}$ and $\operatorname{Ind}_{*}: K^{0}(T V) \rightarrow \mathbb{Z}$ both correspond to the canonical map $K_{0}(V) \rightarrow \mathbb{Z}$ through the Poincaré duality $K_{0}(V) \simeq K^{0}\left(T^{*} V\right)$ and $K_{0}(V) \simeq K^{0}\left(T^{\mathrm{S}} V\right)$. In other words both notions of indices coincide trough the $K K$-equivalence $T V \simeq T^{\mathrm{S}} V$.

We will investigate the case of general stratifications and the proof of an index formula in forthcoming papers. 
The paper is organized as follows. In Section 1 we describe the notion of conical pseudomanifolds and conical bundles. Section 2 reviews general facts about Lie groupoids. Section 3 is devoted to the construction of tangent spaces and tangent groupoids associated to conical pseudomanifolds as well as other deformation groupoids needed in the subsequent sections. Sections 4 and 5 contain the construction of analytical and topological indices, and the last section is devoted to the proof of our topological index theorem for conical pseudomanifolds, that is, the proof of the equality of analytical and topological indices for conical pseudomanifolds.

\section{Cones And stratified Bundles}

We are interested in studying conical pseudomanifolds, which are special examples of stratified pseudomanifolds of depth one [25]. We will use the notations and equivalent descriptions given by A. Verona in [54] or used by J.P. Brasselet, G. Hector and M. Saralegi in [1]. See 29] for a review of the subject.

1.1. Conical pseudomanifolds. If $L$ is a smooth manifold, the cone over $L$ is, by definition, the topological space

$$
c L:=L \times[0,+\infty[/ L \times\{0\} .
$$

Thus $L \times\{0\}$ maps into a single point $c$ of $c L$. We shall refer to $c$ as the singular point of $L$. If $z \in L$ and $t \in[0,+\infty[$ then $[z, t]$ will denote the image of $(z, t)$ in $c L$. We shall denote by

$$
\rho_{c L}: c L \rightarrow\left[0,+\infty\left[, \quad \rho_{c L}([z, t]):=t\right.\right.
$$

the map induced by the second projection and we call it the defining function of the cone.

Definition 1.1. A conical stratification is a triplet $(X, \mathrm{~S}, \mathcal{C})$ where

(i) $X$ is a Hausdorff, locally compact, and secound countable space.

(ii) $\mathrm{S} \subset X$ is a finite set of points, called the singular set of $X$, such that $X^{\circ}:=$ $X \backslash \mathrm{S}$ is a smooth manifold.

(iii) $\mathcal{C}=\left\{\left(\mathcal{N}_{s}, \rho_{s}, L_{s}\right)\right\}_{s \in S}$ is the set of control data, where $\mathcal{N}_{s}$ is an open neighborhood of $s$ in $X$ and $\rho_{s}: \mathcal{N}_{s} \rightarrow[0,+\infty[$ is a surjective continuous map such that $\rho_{s}^{-1}(0)=s$.

(iv) For each $s \in \mathrm{S}$, there exists a homeomorphism $\varphi_{s}: \mathcal{N}_{s} \rightarrow c L_{s}$, called trivialisation map, such that $\rho_{c L_{s}} \circ \varphi_{s}=\rho_{s}$ and such that the induced map $\left.\mathcal{N}_{s} \backslash\{s\} \rightarrow L_{s} \times\right] 0,+\infty\left[\right.$ is a diffeomorphism. Moreover, if $s_{0}, s_{1} \in \mathrm{S}$ then either $\mathcal{N}_{s_{0}} \cap \mathcal{N}_{s_{1}}=\emptyset$ or $s_{0}=s_{1}$.

Let us notice that it follows from the definition that the connected components of $X^{\circ}$ are smooth manifolds. These connected components are called the regular strata of $X$.

Definition 1.2. Two conical stratifications $\left(X, \mathrm{~S}_{X}, \mathcal{C}_{X}\right)$ and $\left(Y, \mathrm{~S}_{Y}, \mathcal{C}_{Y}\right)$ are called isomorphic if there is an homeomorphism $f: X \rightarrow Y$ such that:

(i) $f$ maps $\mathrm{S}_{X}$ onto $\mathrm{S}_{Y}$,

(ii) $f$ restricts to a smooth diffeomorphism $f^{\circ}: X^{\circ} \rightarrow Y^{\circ}$,

(iii) the defining function $\rho_{s}$ of any $s \in \mathrm{S}_{X}$ is equal to $\rho_{f(s)} \circ f$, where $\rho_{f(s)}$ is the defining function of $f(s) \in \mathrm{S}_{Y}$ (in particular $\left.f\left(\mathcal{N}_{s}\right)=\mathcal{N}_{f(s)}\right)$. 
An isomorphism class of conical stratifications will be called a conical pseudomanifold.

In other words, a conical pseudomanifold is a locally compact, metrizable, second countable space $X$ together with a finite set of points $\mathrm{S} \subset X$ such that $X^{\circ}=X \backslash \mathrm{S}$ is a smooth manifold and one can find a set of control data $\mathcal{C}$ such that $(X, S, \mathcal{C})$ is a conical stratification.

Let $M$ be a smooth manifold with boundary $L:=\partial M$. An easy way to construct a conical pseudomanifold is to glue to $M$ the closed cone $\overline{c L}:=L \times[0,1] / L \times\{0\}$ along the boundary.

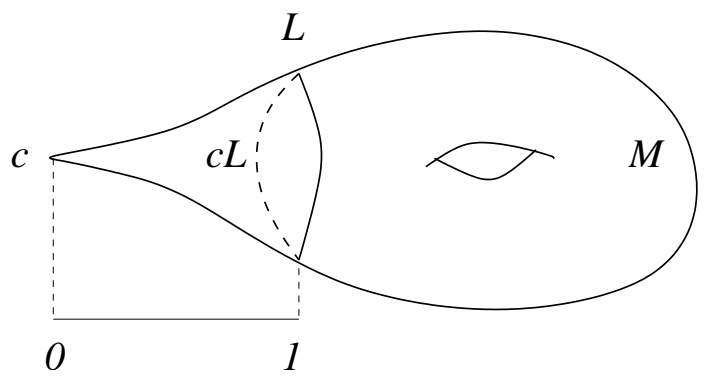

Notice that we do not ask the link $L$ to be connected. For example, if $M$ is a smooth manifold, the space $M \times S^{1} / M \times\{p\}, p \in S^{1}$, is a conical pseudomanifold with $L$ consisting of two disjoint copies of $M$ :

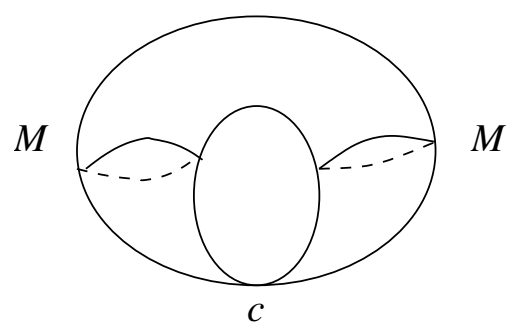

1.2. Conical bundles. We next introduce "conical bundles," a class of spaces not to be confused with vector bundles over conical manifolds. Assume that $L$ is a smooth manifold, $c L$ is the cone over $L, \pi_{\xi}: \xi \rightarrow L$ is a smooth vector bundle over $L$, and $c \xi$ is the cone over $\xi$. We define $\pi: c \xi \rightarrow c L$ by $\pi([z, t])=\left[\pi_{\xi}(z), t\right]$ for $(z, t) \in \xi \times\left[0,+\infty\left[\right.\right.$. The set $(c \xi, \pi)$ is the cone over the vector bundle $\left(\xi, \pi_{\xi}\right)$. Let us notice that the fiber above the singular point of $c L$ is the singular point of $c \xi$. In particular, $c \xi$ is not a vector bundle over $c L$.

Definition 1.3. Let $\left(X, \mathrm{~S}_{X}, \mathcal{C}_{X}\right)$ be a conical stratification. A conical vector bundle $(E, \pi)$ over $X$ is a conical stratification $\left(E, S_{E}, \mathcal{C}_{E}\right)$ together with a continuous surjective map $\pi: E \rightarrow X$ such that:

(1) $\pi$ induces a bijection between the singular sets $S_{E}$ and $S_{X}$.

(2) If $E^{\circ}:=E \backslash \mathrm{S}_{E}$, the restriction $\pi^{\circ}: E^{\circ} \rightarrow X^{\circ}$ is a smooth vector bundle.

(3) The control data $\left\{\mathcal{M}_{z}, \rho_{z}, \xi_{z}\right\}_{z \in \mathrm{S}_{E}}$ of $E$ and $\left\{\mathcal{N}_{s}, \rho_{s}, L_{s}\right\}_{s \in \mathrm{S}_{X}}$ of $X$ satisfies: $\mathcal{M}_{z}=\pi^{-1}\left(\mathcal{N}_{\pi(z)}\right)$ and $\rho_{z}=\rho_{\pi(z)} \circ \pi$. Moreover for $z \in \mathrm{S}_{E}$ and $s=\pi(z) \in$ $\mathrm{S}_{X}$, the restriction $\pi_{z}: \mathcal{M}_{z} \rightarrow \mathcal{N}_{s}$ is a cone over the vector bundle $\xi_{z}$. More 
precisely, we have the following commutative diagram

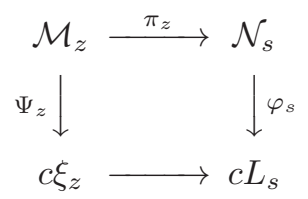

where $\xi_{z} \rightarrow L_{s}$ is a smooth vector bundle over $L_{s}$, the bottom horizontal arrow is the cone over $\xi_{z} \rightarrow L_{s}$ and $\Psi_{z}, \varphi_{s}$ are trivialisation maps.

If $X$ is a conical pseudomanifold, the isomorphism class of a conical vector bundle over a conical stratification $\left(X, \mathrm{~S}_{X}, \mathcal{C}_{X}\right)$ will be call again a conical vector bundle over $X$.

We are interested in conical vector bundles because they allow us to introduce the right notion of tubular neighborhood in the class of conical manifolds.

Let $L$ be a manifold and $c L$ the cone over $L$. For $N \in \mathbb{N}$ large enough, we can find an embedding $j_{L}: L \rightarrow \mathbb{R}^{N}$. Let $\mathcal{V}_{L} \rightarrow L$ be the normal bundle of this embedding. We let $c \mathcal{V}_{L}=\mathcal{V}_{L} \times\left[0,+\infty\left[/ \mathcal{V}_{L} \times\{0\}\right.\right.$ be the cone over $\mathcal{V}_{L}$; it is a conical vector bundle over $c L$.

Now, let $X=(X, \mathrm{~S}, \mathcal{C})$ be a conical stratification and suppose, for simplicity, that $X$ has only one singularity. Namely, the sets $S=\{c\}$ and $\mathcal{C}=\{(\mathcal{N}, \rho, L)\}$ consist of a single element each, where $\mathcal{N}$ is a cone over $L$ and $\varphi: \mathcal{N} \rightarrow c L$ a trivialisation map. For $N \in \mathbb{N}$ large enough, one can find an embedding $j: X^{\circ}=X \backslash\{c\} \rightarrow$ $\mathbb{R}^{N}=\mathbb{R}^{N-1} \times \mathbb{R}$, such that its restriction to $\left.\mathcal{N} \backslash\{c\} \simeq L \times\right] 0,+\infty[$ satisfies

$$
\left.j \circ \varphi^{-1}\right|_{L \times] 0,+\infty[}=j_{L} \times \mathrm{Id},
$$

for an embedding $j_{L}: L \rightarrow \mathbb{R}^{N-1}$. Let $\mathcal{V}_{L} \rightarrow L$ be the normal bundle of the embedding $j_{L}$ and $\mathcal{V} \rightarrow X^{\circ}$ be the normal bundle of the embedding $j$. Then we can identify the restriction $\left.\left.\mathcal{V}\right|_{\mathcal{N} \backslash\{c\}}=\mathcal{V}_{L} \times\right] 0, \infty[$. We define the conical manifold $\mathcal{W}=c \mathcal{V}_{L} \cup_{T \varphi} \mathcal{V}$ by glueing with $T \varphi$ the restriction of $\mathcal{V}$ over $\mathcal{N}_{c} \backslash\{c\}$ with $c \mathcal{V}_{L} \backslash\{c\}$. The conical manifold $\mathcal{W}$ is a conical vector bundle over $X$. It follows that $\mathcal{W}$ is a sub-stratified pseudomanifold of $\left(\mathbb{R}^{N}\right)^{c}:=c \mathbb{R}^{N-1}$, the cone over $\mathbb{R}^{N-1}$. We will say that $\mathcal{W}$ is the tubular neighborhood of the embedding of $X$ in $\left(\mathbb{R}^{N}\right)^{c}$.

\section{Lie GROUPOIDS AND THEIR LIE ALGEBROIDS}

We refer to 49, 12, 34 for the classical definitions and construction related to groupoids and their Lie algebroids.

2.1. Lie groupoids. Groupoids, and especially differentiable groupoids will play an important role in what follows, so we recall the basic definitions and results needed for this paper. Recall first that a groupoid is a small category in which every morphism is an isomorphism.

Let us make the notion of a groupoid more explicit. Thus, a groupoid $\mathcal{G}$ is a pair $\left(\mathcal{G}^{(0)}, \mathcal{G}^{(1)}\right)$ of sets together with structural morphisms $u: \mathcal{G}^{(0)} \rightarrow \mathcal{G}^{(1)}$, $s, r: \mathcal{G}^{(1)} \rightarrow \mathcal{G}^{(0)}, \iota: \mathcal{G}^{(1)} \rightarrow \mathcal{G}^{(1)}$, and, especially, the multiplication $\mu$ which is defined for pairs $(g, h) \in \mathcal{G}^{(1)} \times \mathcal{G}^{(1)}$ such that $s(g)=r(h)$. Here, the set $\mathcal{G}^{(0)}$ denotes the set of objects (or units) of the groupoid, whereas the set $\mathcal{G}^{(1)}$ denotes the set of morphisms of $\mathcal{G}$. Each object of $\mathcal{G}$ can be identified with a morphism of $\mathcal{G}$, the identity morphism of that object, which leads to an injective map $u: \mathcal{G}^{(0)} \rightarrow \mathcal{G}$. Each morphism $g \in \mathcal{G}$ has a "source" and a "range." We shall denote by $s(g)$ the 
source of $g$ and by $r(g)$ the range of $g$. The inverse of a morphism $g$ is denoted by $g^{-1}=\iota(g)$. The structural maps satisfy the following properties:

(i) $r(g h)=r(g)$ and $s(g h)=s(h)$, for any pair $g$, $h$ satisfying $s(g)=r(h)$,

(ii) $s(u(x))=r(u(x))=x, u(r(g)) g=g, g u(s(g))=g$,

(iii) $r\left(g^{-1}\right)=s(g), s\left(g^{-1}\right)=r(g), g g^{-1}=u(r(g))$, and $g^{-1} g=u(s(g))$,

(iv) the partially defined multiplication $\mu$ is associative.

We shall need groupoids with smooth structures.

Definition 2.1. A Lie groupoid is a groupoid

$$
\mathcal{G}=\left(\mathcal{G}^{(0)}, \mathcal{G}^{(1)}, s, r, \mu, u, \iota\right)
$$

such that $\mathcal{G}^{(0)}$ and $\mathcal{G}^{(1)}$ are manifolds with corners, the structural maps $s, r, \mu, u$, and $\iota$ are differentiable, the domain map $s$ is a submersion and $\mathcal{G}_{x}:=s^{-1}(x)$, $x \in M$, are all Hausdorff manifolds without corners.

The term "differentiable groupoid" was used in the past instead of "Lie groupoid," whereas "Lie groupoid" had a more restricted meaning [34. The usage has changed however more recently, and our definition reflects this change.

An example of a Lie groupoid that will be used repeatedly below is that of pair groupoid, which we now define. Let $M$ be a smooth manifold. We let $\mathcal{G}^{(0)}=M$, $\mathcal{G}^{(1)}=M \times M, s(x, y)=y, r(x, y)=x,(x, y)(y, z)=(x, z)$, and embedding $u(x)=(x, x)$. The inverse is $\iota(x, y)=(y, x)$.

The infinitesimal object associated to a Lie groupoid is its "Lie algebroid," which we define next.

Definition 2.2. A Lie algebroid $A$ over a manifold $M$ is a vector bundle $A \rightarrow M$, together with a Lie algebra structure on the space $\Gamma(A)$ of smooth sections of $A$ and a bundle map $\varrho: A \rightarrow T M$ whose extension to sections of these bundles satisfies

(i) $\varrho([X, Y])=[\varrho(X), \varrho(Y)]$, and

(ii) $[X, f Y]=f[X, Y]+(\varrho(X) f) Y$,

for any smooth sections $X$ and $Y$ of $A$ and any smooth function $f$ on $M$.

The map $\varrho$ is called the anchor map of $A$. Note that we allow the base $M$ in the definition above to be a manifold with corners.

The Lie algebroid associated to a differentiable groupoid $\mathcal{G}$ is defined as follows 34. The vertical tangent bundle (along the fibers of $s$ ) of a differentiable groupoid $\mathcal{G}$ is, as usual,

$$
T_{\text {vert }} \mathcal{G}=\operatorname{ker} s_{*}=\bigcup_{x \in M} T \mathcal{G}_{x} \subset T \mathcal{G} .
$$

Then $A(\mathcal{G}):=\left.T_{\text {vert }} \mathcal{G}\right|_{M}$, the restriction of the $s$-vertical tangent bundle to the set of units, defines the vector bundle structure on $A(\mathcal{G})$.

We now construct the bracket defining the Lie algebra structure on $\Gamma(A(\mathcal{G}))$. The right translation by an arrow $g \in \mathcal{G}$ defines a diffeomorphism

$$
R_{g}: \mathcal{G}_{r(g)} \ni g^{\prime} \longmapsto g^{\prime} g \in \mathcal{G}_{d(g)} .
$$

A vector field $X$ on $\mathcal{G}$ is called $s$-vertical if $s_{*}(X(g))=0$ for all $g$. The $s$-vertical vector fields are precisely the vector fields on $\mathcal{G}$ that can be restricted to vector fields on the submanifolds $\mathcal{G}_{x}$. It makes sense then to consider right-invariant vector 
fields on $\mathcal{G}$. It is not difficult to see that the sections of $A(\mathcal{G})$ are in one-to-one correspondence with $s$-vertical, right-invariant vector fields on $\mathcal{G}$.

The Lie bracket $[X, Y]$ of two $s$-vertical, right-invariant vector fields $X$ and $Y$ is also $s$-vertical and right-invariant, and hence the Lie bracket induces a Lie algebra structure on the sections of $A(\mathcal{G})$. To define the action of the sections of $A(\mathcal{G})$ on functions on $M$, let us observe that the right invariance property makes sense also for functions on $\mathcal{G}$, and that $\mathcal{C}^{\infty}(M)$ may be identified with the subspace of smooth, right-invariant functions on $\mathcal{G}$. If $X$ is a right-invariant vector field on $\mathcal{G}$ and $f$ is a right-invariant function on $\mathcal{G}$, then $X(f)$ will still be a right invariant function. This identifies the action of $\Gamma(A(\mathcal{G}))$ on $\mathcal{C}^{\infty}(M)$.

2.2. Pull back groupoids. Let $G \rightrightarrows M$ be a groupoid with source $s$ and range $r$. If $f: N \rightarrow M$ is a surjective map, the pull back groupoid ${ }^{*} f^{*}(G) \rightrightarrows N$ of $G$ by $f$ is by definition the set

$$
{ }^{*} f^{*}(G):=\{(x, \gamma, y) \in N \times G \times N \mid r(\gamma)=f(x), s(\gamma)=f(y)\}
$$

with the structural morphisms given by

(1) the unit map $x \mapsto(x, f(x), x)$,

(2) the source map $(x, \gamma, y) \mapsto y$ and range map $(x, \gamma, y) \mapsto x$,

(3) the product $(x, \gamma, y)(y, \eta, z)=(x, \gamma \eta, z)$ and inverse $(x, \gamma, y)^{-1}=\left(y, \gamma^{-1}, x\right)$.

The results of [4] apply to show that the groupoids $G$ and ${ }^{*} f^{*}(G)$ are Morita equivalent.

Let us assume for the rest of this subsection that $G$ is a smooth groupoid and that $f$ is a surjective submersion, then ${ }^{*} f^{*}(G)$ is also a Lie groupoid. Let $(\mathcal{A}(G), q,[]$, be the Lie algebroid of $G$ (which is defined since $G$ is smooth). Recall that $q$ : $\mathcal{A}(G) \rightarrow T M$ is the anchor map. Let $\left(\mathcal{A}\left({ }^{*} f^{*}(G)\right), p,[],\right)$ be the Lie algebroid of ${ }^{*} f^{*}(G)$ and $T f: T N \rightarrow T M$ be the differential of $f$. Then we claim that there exists an isomorphism

$$
\mathcal{A}\left({ }^{*} f^{*}(G)\right) \simeq\{(V, U) \in T N \times \mathcal{A}(G) \mid T f(V)=q(U) \in T M\}
$$

under which the anchor map $p: \mathcal{A}\left({ }^{*} f^{*}(G)\right) \rightarrow T N$ identifies with the projection $T N \times \mathcal{A}(G) \rightarrow T N$. In particular, if $(U, V) \in \mathcal{A}\left({ }^{*} f^{*}(G)\right)$ with $U \in T_{x} N$ and $V \in \mathcal{A}_{y}(G)$, then $y=f(x)$.

2.3. Quasi-graphoid and almost injective Lie algebroid. Our Lie groupoids arise mostly as Lie groupoids with a given Lie algebroid. This is because often in Analysis, one is given the set of derivations (differential operators), which forms a Lie algebra under the commutator. The groupoids are then used to "quantize" the given Lie algebra of vector fields to algebra of pseudodifferential operators 1] 37, 39, 48. This has motivated several works on the integration of Lie algebroids 19, 20, 47. We recall here some useful results of the first named author 20] on the integration of some Lie algebroids. See also [19, 34, 47.

Proposition 2.3. Let $G \underset{r}{\stackrel{s}{\rightrightarrows}} M$ be a Lie groupoid over the manifold $M$. Let us denote by $s$ its domain map, by $r$ its range map, and by $u: M \longrightarrow G$ its unit map. The two following assertions are equivalent:

1. If $\nu: V \longrightarrow G$ is a local section of $s$ then $r \circ \nu=1_{V}$ if, and only if, $\nu=\left.u\right|_{V}$.

2. If $N$ is a manifold, $f$ and $g$ are two smooth maps from $N$ to $G$ such that:

(i) $s \circ f=s \circ g$ and $r \circ f=r \circ g$, 
(ii) one of the following maps $s \circ f$ and $r \circ f$ is a submersion, then $f=g$.

Definition 2.4. A Lie groupoid that satisfies one of the two equivalent properties of Proposition 2.3 will be called a quasi-graphoid.

Suppose that $G \rightrightarrows M$ is a quasi-graphoid and denote by $\mathcal{A} G=(p: \mathcal{A} G \rightarrow$ $\left.T M,[,]_{\mathcal{A}}\right)$ its Lie algebroid. A direct consequence of the previous definition is that the anchor $p$ of $\mathcal{A} G$ is injective when restricted to a dense open subset of the base space $M$. In other words the anchor $p$ induces an injective morphism $\tilde{p}$ from the set of smooth local sections of $\mathcal{A} G$ onto the set of smooth local tangent vector fields over $M$. In this situation we say that the Lie algebroid $\mathcal{A} G$ is almost injective.

A less obvious remarkable property of a quasi-graphoid is that its $s$-connected component is determined by its infinitesimal structure. Precisely:

Proposition 2.5. 20] Two s-connected quasi-graphoids having the same space of units are isomorphic if, and only if, their Lie algebroids are isomorphic.

Note that we are not requiring the groupoids in the above proposition to be $s$-simply connected. The main result of [20] is the following:

Theorem 2.6. Every almost injective Lie algebroid is integrable by an s-connected quasi-graphoid (uniquely by the above proposition).

Finally, let $\mathcal{A}$ be a smooth vector bundle over a manifold $M$ and $p: \mathcal{A} \rightarrow T M$ a morphism. We denote by $\tilde{p}$ the map induced by $p$ from the set of smooth local section of $\mathcal{A}$ to the set of smooth local vector fields on $M$. Notice that if $\tilde{p}$ is injective then $\mathcal{A}$ can be equipped with a Lie algebroid structure over $M$ with anchor $p$ if, and only if, the image of $\tilde{p}$ is stable under the Lie bracket.

Examples 2.7. Regular foliation: A smooth regular foliation $\mathcal{F}$ on a manifold $M$ determines an integrable subbundle $F$ of $T M$. Such a subbundle is an (almost) injective Lie algebroid over $M$. The holonomy groupoid of $\mathcal{F}$ is the $s$-connected quasi-graphoid which integrates $F$ [56].

Tangent groupoid: One typical example of a quasi-graphoid is the tangent groupoid of A. Connes [16. Let us denote by $A \sqcup B$ the disjoint union of the sets $A$ and $B$. If $M$ is a smooth manifold, the tangent groupoid of $M$ is the disjoint union

$$
\left.\left.\mathcal{G}_{M}^{t}=T M \times\{0\} \sqcup M \times M \times\right] 0,1\right] \rightrightarrows M \times[0,1] .
$$

In order to equip $\mathcal{G}_{M}^{t}$ with a smooth structure, we choose a riemannian metric on $M$ and we require that the map

$$
\begin{array}{cl}
V \subset T M \times[0,1] & \longrightarrow \\
(x, V, t) & \mapsto\left\{\begin{array}{c}
(x, V, 0) \text { if } t=0 \\
\left(x, \exp _{x}(-t V), t\right) \text { if } t \neq 0
\end{array}\right.
\end{array}
$$

be a smooth diffeomorphism onto its image, where $V$ is open in $T M \times[0,1]$ and contains $T M \times\{0\}$. The tangent groupoid of $M$ is the $s$-connected quasi-graphoid which integrates the almost injective Lie algebroid:

$$
\begin{array}{ccc}
p_{M}^{t}: \mathcal{A G}_{M}^{t}=T M \times[0,1] & \longrightarrow & T(M \times[0,1]) \simeq T M \times T[0,1] \\
(x, V, t) & \mapsto & (x, t V ; t, 0)
\end{array}
$$


2.4. Deformation of quasi-graphoids. In this paper, we will encounter deformation groupoids. The previous results give easy aguments to get sure that these deformation groupoids can be equipped with a smooth structure. For example, let $G_{i} \rightrightarrows M, i=1,2$, be two $s$-connected quasi-graphoids over the manifold $M$ and let $\mathcal{A} G_{i}=\left(p_{i}: \mathcal{A} G_{i} \rightarrow T M,[,]_{\mathcal{A}_{i}}\right)$ be the corresponding Lie algebroid. Suppose that:

- The bundles $\mathcal{A} G_{1}$ and $\mathcal{A} G_{2}$ are isomorphic,

- There is a morphism $p: \mathcal{A}:=\mathcal{A} G_{1} \times[0,1] \rightarrow T M \times T([0,1])$ of the form:

$$
p(V, 0)=\left(p_{1}(V) ; 0,0\right) \text { and } p(V, t)=\left(p_{2} \circ \Phi(V, t) ; t, 0\right) \text { if } t \neq 0,
$$

where $\left.\left.\left.\left.\Phi: \mathcal{A} G_{1} \times\right] 0,1\right] \rightarrow \mathcal{A} G_{2} \times\right] 0,1\right]$ is an isomorphism of bundles over $M \times] 0,1]$. Moreover the image of $\tilde{p}$ is stable under the Lie bracket.

In this situation, $\mathcal{A}$ is an almost injective Lie algebroid that can be integrated by the groupoid $\left.\left.H=G_{1} \times\{0\} \cup G_{2} \times\right] 0,1\right] \rightrightarrows M \times[0,1]$. In particular, there is a smooth structure on $H$ compatible with the smooth structure on $G_{1}$ and $G_{2}$.

\section{A NON-COMMUtATIVE TANGENT SPACE FOR CONICAL PSEUdOMANIFOlDS}

In order to obtain an Atiyah-Singer type topological index theorem for our conical pseudomanifold $X$, we introduce in this chapter a suitable notion of tangent space to $X$ and a suitable normal space to an embedding of $X$ in $\mathbb{R}^{N+1}$ that sends the singular point to 0 and $X^{\circ}$ to $\left\{x_{1}>0\right\}$.

3.1. The S-tangent space and the tangent groupoid of a conical space. We recall here a construction from 21] that associates to a conical pseudomanifold $X$ a groupoid $T^{\mathrm{S}} X$ that is a replacement of the notion of tangent space of $X$ (for the purpose of studying $K$-theory) in the sense the $C^{*}$-algebras $C^{*}\left(T^{\mathcal{S}} X\right)$ and $C(X)$ are $K$-dual [21].

Let $(X, \mathrm{~S}, \mathcal{C})$ be a conical pseudomanifold. Without loss of generality, we can assume that $X$ has only one singular point. Thus $\mathrm{S}=\{c\}$ is a single point and $\mathcal{C}=$ $\{(\mathcal{N}, \rho, L)\}$, where $\mathcal{N} \simeq c L$ is a cone over $L$ and $\rho$ is the defining function of the cone. We set $\rho=+\infty$ outside $\mathcal{N}$. We let $X^{\circ}=X \backslash\{c\}$. Recall that $X^{\circ}$ is a smooth manifold. We denote by $O_{X}$ the open set $O_{X}=\left\{z \in X^{\circ} \mid \rho(z)<1\right\}$.

At the level of sets, the $\mathrm{S}$-tangent space of $X$ is the groupoid:

$$
T^{\mathrm{S}} X:=\left.T X^{\circ}\right|_{X \circ} \backslash O_{X} \sqcup O_{X} \times O_{X} \rightrightarrows X^{\circ} .
$$

Here, the groupoid $\left.T X^{\circ}\right|_{X^{\circ} \backslash O_{X}} \rightrightarrows X^{\circ} \backslash O_{X}$ is the usual tangent vector bundle $T X^{\circ}$ of $X^{\circ}$ restricted to the closed subset $X^{\circ} \backslash O_{X}=\left\{z \in X^{\circ} \mid \rho(z) \geq 1\right\}$. The groupoid $O_{X} \times O_{X} \rightrightarrows O_{X}$ is the pair groupoid over $O_{X}$.

The tangent groupoid of $X$ is, as in the regular case [16, a deformation of its "tangent space" to the pair groupoid over its units:

$$
\left.\left.\mathcal{G}_{X}^{t}:=T^{\mathrm{S}} X \times\{0\} \sqcup X^{\circ} \times X^{\circ} \times\right] 0,1\right] \rightrightarrows X^{\circ} \times[0,1] .
$$

Here, the groupoid $\left.\left.\left.\left.X^{\circ} \times X^{\circ} \times\right] 0,1\right] \rightrightarrows X^{\circ} \times\right] 0,1\right]$ is the product of the pair groupoid on $X^{\circ}$ with the set $\left.] 0,1\right]$.

In order to equip $\mathcal{G}_{X}^{t}$, and so $T^{\mathrm{S}} X$, with a smooth structure we have to choose a glueing function. First choose a positive smooth map $\tau: \mathbb{R} \rightarrow \mathbb{R}$ such that $\tau\left(\left[0,+\infty[)=[0,1], \tau^{-1}(0)=\left[1,+\infty\left[\right.\right.\right.\right.$ and $\tau^{\prime}(t) \neq 0$ for $t<1$. We denote by 
$\tau_{X}: X \rightarrow \mathbb{R}$ the map which assigns $\tau(\rho(x))$ to $x \in X^{\circ} \cap \mathcal{N}$ and 0 elsewhere. Thus $\tau_{X}\left(X^{\circ}\right)=\left[0,1\left[, \tau_{X}\right.\right.$ restricted to $O_{X}=\{z \in \mathcal{N} \mid 0<\rho(z)<1\}$ is a submersion and $\tau_{X}^{-1}(0)=X^{\circ} \backslash O_{X}$.

Proposition 3.1. 21] There is a unique structure of Lie groupoid on $\mathcal{G}_{X}^{t}$ such that its Lie algebroid is the bundle $T X^{\circ} \times[0,1]$ whith anchor $p:(x, V, t) \in T X^{\circ} \times[0,1] \mapsto$ $\left(x,\left(t+\tau_{X}^{2}(x)\right) V ; t, 0\right) \in T X^{\circ} \times T[0,1]$.

Let us notice that the map $p$ is injective when restricted to $\left.\left.X^{\circ} \times\right] 0,1\right]$, which is a dense open subset of $X^{\circ} \times[0,1]$. Thus there exists one, and only one, structure of (almost injective) Lie algebroid on $T X^{\circ} \times[0,1]$ with $p$ as anchor since the family of local vector fields on $X^{\circ}$ induced by the image by $p$ of local sections of $T X^{\circ} \times[0,1]$ is stable under the Lie bracket. We know from [20, 47] that such a Lie algebroid is integrable. Moreover, according to theorem 2.6 there is a unique Lie groupoid which integrates this algebroid and restricts over $\left.\left.X^{\circ} \times\right] 0,1\right]$ to $\left.\left.X^{\circ} \times X^{\circ} \times\right] 0,1\right] \rightrightarrows$ $\left.\left.X^{\circ} \times\right] 0,1\right]$.

Let us give an alternative proof of the previous proposition.

Proof. Recall that the (classical) tangent groupoid of $X^{\circ}$ is

$$
\left.\left.\mathcal{G}_{X^{\circ}}^{t}=T X^{\circ} \times\{0\} \sqcup X^{\circ} \times X^{\circ} \times\right] 0,1\right] \rightrightarrows X^{\circ} \times[0,1]
$$

and that its Lie algebroid is the bundle $T X^{\circ} \times[0,1]$ over $X^{\circ} \times[0,1]$ with anchor $(x, V, t) \in T X^{\circ} \times[0,1] \mapsto(x, t V, t, 0) \in T X^{\circ} \times T[0,1]$. Similary, one can equip the groupoid $H=T X^{\circ} \times\{(0,0)\} \sqcup X^{\circ} \times X^{\circ} \times[0,1]^{2} \backslash\{(0,0)\}$ with a unique smooth structure such that its Lie algebroid is the bundle $T X^{\circ} \times[0,1]^{2}$ with anchor the map

$$
\begin{array}{ccc}
p: \mathcal{A}=T \mathcal{N}_{1} \times[0,1] \times[0,1] & \rightarrow & T \mathcal{N}_{1} \times T([0,1]) \times T([0,1]) \\
(x, V, t, l) & \mapsto & (x,(t+l) V ; t, 0 ; l, 0)
\end{array}
$$

Let $\delta: H \rightarrow \mathbb{R}$ be the map which sends any $\gamma \in H$ with source $s(\gamma)=(y, t, l)$ and range $r(\gamma)=(x, t, l)$ to $\delta(\gamma)=l-\tau_{X}(x) \tau_{X}(y)$. One can check that $\delta$ is a smooth submersion, so $H_{\delta}:=\delta^{-1}(0)$ is a submanifold of $H$. Moreover $H_{\delta}:=\delta^{-1}(0)$ inherits from $H$ a structure of Lie groupoid over $X^{\circ} \times[0,1]$ whose Lie algebroid is given by

$$
\begin{array}{ccc}
T X^{\circ} \times[0,1] & \rightarrow & T X^{\circ} \times T([0,1]) \\
(x, V, t) & \mapsto & \left(x,\left(t+\tau_{X}^{2}(x)\right) V ; t, 0\right)
\end{array}
$$

The groupoid $H_{\delta}$ is (obviously isomorphic) to $\mathcal{G}_{X}^{t}$.

We now introduce the tangent groupoid of a stratified pseudomanifold.

Definition 3.2. The groupoid $\mathcal{G}_{X}^{t}$ equipped with the smooth structure associated with a glueing function $\tau$ as above is called a tangent groupoid of the stratified pseudomanifold $(X, \mathrm{~S}, \mathcal{C})$. The corresponding $\mathrm{S}$-tangent space is the groupoid $\left.T^{\mathrm{S}} X \simeq \mathcal{G}_{X}^{t}\right|_{X \circ \times\{0\}}$ equipped with the induced smooth structure.

Remarks 3.3. We will need the following remarks. See 21 for a proof.

(i) If $X$ has more than one singular point, we let, for any $s \in \mathrm{S}$,

$$
O_{s}:=\left\{z \in X^{\circ} \cap \mathcal{N}_{s} \mid \rho_{s}(z)<1\right\},
$$

and we define $O=\sqcup_{s \in \mathrm{S}} O_{s}$. The S-tangent space to $X$ is then

$$
T^{\mathrm{S}} X:=\left.T X^{\circ}\right|_{X^{\circ} \backslash O} \sqcup_{s \in \mathrm{S}} O_{s} \times O_{s} \rightrightarrows X^{\circ},
$$

with the analogous smooth structure. 
(ii) The orbit space of $T^{\mathrm{S}} X$ is topologicaly equivalent to $X$ : there is a canonical isomorphism between the algebras $C(X)$ and $C\left(X / T^{\mathrm{S}} X\right)$.

(iii) The tangent groupoid and the $\mathrm{S}$-tangent space depend on the glueing. Nevertheless the $K$-theory of the $C^{*}$-algebras $C^{*}\left(\mathcal{G}_{X}^{t}\right)$ and $C^{*}\left(T^{\mathrm{S}} X\right)$ do not.

(iv) The groupoid $T^{\mathrm{S}} X$ is a continuous field of amenable groupoids parametrized by $X$, thus $T^{\mathrm{S}} X$ is amenable as well. It follows that $\mathcal{G}_{X}^{t}$ is also amenable as a continuous field of amenable groupoids parametrised by $[0,1]$. Hence the reduced and maximal $C^{*}$-algebras of $T^{S} X$ and of $\mathcal{G}_{X}^{t}$ are equal and they are nuclear.

Examples 3.4. Here are two basic examples.

(i) When $X$ is a smooth manifold, that is $X_{0}=\emptyset$ and $X^{\circ}=X$, the previous construction gives rise to the usual tangent groupoid

$$
\left.\left.\mathcal{G}_{X}^{t}=T X \times\{0\} \sqcup X \times X \times\right] 0,1\right] \rightrightarrows X \times[0,1] .
$$

Moreover, $T^{\mathrm{S}} X=T X \rightrightarrows X$ is the usual tangent space.

(ii) Let $L$ be a manifold and consider the (trivial) cone $c L=L \times[0,+\infty[/ L \times\{0\}$ over $L$. In this situation $\left.X^{\circ}=L \times\right] 0,+\infty\left[, O_{X}=L \times\right] 0,1[$ and

$$
T^{\mathrm{S}} X=T(L \times[1,+\infty[) \sqcup \underbrace{L \times] 0,1[\times L \times] 0,1[}_{\text {the pair groupoid }} \rightrightarrows L \times] 0,+\infty[,
$$

where $T(L \times[1,+\infty[)$ denotes the restriction to $L \times[1,+\infty[$ of the tangent space $T(L \times \mathbb{R})$. The general case is always locally of this form.

3.2. The deformation groupoid of a conical vector bundle. Let $\left(E, S_{E}, \mathcal{C}_{E}\right)$ be a conical vector bundle over $\left(X, \mathrm{~S}_{X}, \mathcal{C}_{X}\right)$ and denote by $\pi: E \rightarrow X$ the corresponding projection. From the definition, $\pi$ restricts to a smooth vector bundle map $\pi^{\circ}: E^{\circ} \rightarrow X^{\circ}$. We let $\pi_{[0,1]}=\pi^{\circ} \times i d: E^{\circ} \times[0,1] \rightarrow X^{\circ} \times[0,1]$.

We consider the tangent groupoids $\mathcal{G}_{X}^{t} \rightrightarrows X^{\circ}$ for $X$ and $\mathcal{G}_{E}^{t} \rightrightarrows E^{\circ}$ for $E$ equipped with a smooth structure constructed using the same glueing fonction $\tau$ (in particular $\left.\tau_{X} \circ \pi=\tau_{E}\right)$. We denote by ${ }^{*} \pi_{[0,1]}^{*}\left(\mathcal{G}_{X}^{t}\right) \rightrightarrows E^{\circ} \times[0,1]$ the pull back of $\mathcal{G}_{X}^{t}$ by $\pi_{[0,1]}$.

Our next goal is to associate to the conical vector bundle $E$ a deformation groupoid $\mathcal{T}_{E}^{t}$ using ${ }^{*} \pi_{[0,1]}^{*}\left(\mathcal{G}_{X}^{t}\right)$ to $\mathcal{G}_{E}^{t}$. More precisely, we define:

$$
\left.\left.\mathcal{T}_{E}^{t}:=\mathcal{G}_{E}^{t} \times\{0\} \sqcup^{*} \pi_{[0,1]}^{*}\left(\mathcal{G}_{X}^{t}\right) \times\right] 0,1\right] \rightrightarrows E^{\circ} \times[0,1] \times[0,1] .
$$

In order to equip $\mathcal{T}_{E}^{t}$ with a smooth structure, we first choose a smooth projection $P: T E^{\circ} \rightarrow \operatorname{Ker}(T \pi)$.

A simple calculation shows that the Lie algebroid of ${ }^{*} \pi_{[0,1]}^{*}\left(\mathcal{G}_{X}^{t}\right)$ is isomorphic to the bundle $T E^{\circ} \times[0,1]$ endowed with the almost injective anchor map

$$
(x, V, t) \mapsto\left(x, P(x, V)+\left(t+\tau_{E}(x)^{2}\right)(V-P(x, V)) ; t, 0\right) .
$$

We consider the bundle $\mathcal{A}=T E^{\circ} \times[0,1] \times[0,1]$ over $E^{\circ} \times[0,1] \times[0,1]$ and the almost injective morphism:

$$
\begin{array}{ccc}
p: \mathcal{A}=T E^{\circ} \times[0,1] \times[0,1] & \rightarrow T X^{\circ} \times T[0,1] \times T[0,1] \\
(x, V, t, l) & \mapsto\left(x,\left(t+\tau_{E}^{2}(x)\right) V+l P(x, V)\right) .
\end{array}
$$

The image of $\tilde{p}$ is stable under the Lie bracket, thus $\mathcal{A}$ is an almost injective Lie algebroid. Moreover, the restriction of $\mathcal{A}$ to $E^{\circ} \times[0,1] \times\{0\}$ is the Lie algebroid of $\mathcal{G}_{E}^{t}$ and its restriction to $\left.\left.E^{\circ} \times[0,1] \times\right] 0,1\right]$ is isomorphic to the Lie algebroid of 
$\left.\left.{ }^{*} \pi_{[0,1]}^{*}\left(\mathcal{G}_{X}^{t}\right) \times\right] 0,1\right]$. Thus $\mathcal{A}$ can be integrated by $\mathcal{T}_{E}^{t}$. In particular, $\mathcal{T}_{E}^{t}$ is a smooth groupoid. In conclusion, the restriction of $\mathcal{T}_{E}^{t}$ to $E^{\circ} \times\{0\} \times[0,1]$ leads to a Lie groupoid:

$$
\left.\left.\mathcal{H}_{E}=T^{S} E \times\{0\} \sqcup^{*} \pi^{*}\left(T^{S} X\right) \times\right] 0,1\right] \rightrightarrows E^{\circ} \times[0,1],
$$

called a Thom groupoid associated to the conical vector bundle $E$ over $X$.

The following example explains what these constructions become if there are no singularities.

Example 3.5. Suppose that $p: E \rightarrow M$ is a smooth vector bundle over the smooth manifold $M$. Then $\left.\left.\mathrm{S}_{E}=\mathrm{S}_{M}=\emptyset, \mathcal{G}_{E}^{t}=T E \times\{0\} \sqcup E \times E \times\right] 0,1\right] \rightrightarrows E \times[0,1]$ and $\left.\left.\mathcal{G}_{M}^{t}=T M \times\{0\} \sqcup M \times M \times\right] 0,1\right] \rightrightarrows M \times[0,1]$ are the usual tangent groupoids. In these examples associated to a smooth vector bundle, $\tau_{E}$ is the zero map. The groupoid $\mathcal{T}_{E}^{t}$ will then be given by

$\left.\left.\left.\left.\mathcal{T}_{E}^{t}=T E \times\{0\} \times\{0\} \sqcup^{*} p^{*}(T M) \times\{0\} \times\right] 0,1\right] \sqcup E \times E \times\right] 0,1\right] \times[0,1] \rightrightarrows E \times[0,1] \times[0,1]$ and is smooth. Similarly, the Thom groupoid will be given by: $\mathcal{H}_{E}:=T E \times\{0\} \sqcup$ $\left.\left.{ }^{*} p^{*}(T M) \times\right] 0,1\right] \rightrightarrows E \times[0,1]$.

We now return to the general case of a conical vector bundle.

Remark 3.6. The groupoids $\mathcal{T}_{E}$ and $\mathcal{H}_{E}$ are continuous fields of amenable groupoids parametrized by $[0,1]$. Thus they are amenable, their reduced and maximal $C^{*}$ algebras are equal, and are nuclear.

\section{The anALYTICAL InDEX}

Let $X$ be a conical pseudomanifold, and let

$$
\left.\left.\mathcal{G}_{X}^{t}=X^{\circ} \times X^{\circ} \times\right] 0,1\right] \sqcup T^{\mathrm{S}} X \times\{0\} \rightrightarrows X^{\circ} \times[0,1]
$$

be the tangent groupoid (unique up to isomorphism) for $X$ for a given glueing function. Also, let $T^{\mathrm{S}} X \rightrightarrows X^{\circ}$ be the corresponding S-tangent space.

Since the groupoid $\mathcal{G}_{X}^{t}$ is a deformation groupoid of amenable groupoids, it defines a $K K$-element 21] 27]. More precisely, let

$$
e_{1}: C^{*}\left(\mathcal{G}_{X}^{t}\right) \rightarrow C^{*}\left(\left.\mathcal{G}_{X}^{t}\right|_{X^{\circ} \times\{1\}}\right)=\mathcal{K}\left(L^{2}\left(X^{\circ}\right)\right)
$$

be the evaluation at 1 and let $\left[e_{1}\right] \in K K\left(C^{*}\left(\mathcal{G}_{X}^{t}\right), \mathcal{K}\left(L^{2}\left(X^{\circ}\right)\right)\right.$ the element defined by $e_{1}$ in Kasparov's bivariant $K$-theory. Similarly, the evaluation at 0 defines a morphism $e_{0}: C^{*}\left(\mathcal{G}_{X}^{t}\right) \rightarrow C^{*}\left(\left.\mathcal{G}_{X}^{t}\right|_{X^{\circ} \times\{0\}}\right)=C^{*}\left(T^{\mathrm{S}} X\right)$ and then an element $\left[e_{0}\right] \in K K\left(C^{*}\left(\mathcal{G}_{X}^{t}\right), C^{*}\left(T^{\mathrm{S}} X\right)\right)$. The kernel of $e_{0}$ is contractible and so $e_{0}$ is $K K$ invertible. We let:

$$
\widetilde{\partial}=\left[e_{0}\right]^{-1} \otimes\left[e_{1}\right] \in K K\left(C^{*}\left(T^{S} X\right), \mathcal{K}\right),
$$

be the Kasparov product over $C^{*}\left(\mathcal{G}_{X}^{t}\right)$ of $\left[e_{1}\right]$ and the $K$-inverse of $\left[e_{0}\right]$. Take $b$ to be a generator of $K K(\mathcal{K}, \mathbb{C}) \simeq \mathbb{Z}$. We set $\partial=\widetilde{\partial} \otimes b$. The element $\partial$ belongs to $K K\left(C^{*}\left(T^{\mathrm{S}} X\right), \mathbb{C}\right)$.

Definition 4.1. The map $\left(e_{0}\right)_{*}: K_{0}\left(C^{*}\left(\mathcal{G}_{X}^{t}\right)\right) \rightarrow K_{0}\left(C^{*}\left(T^{S} X\right)\right)$ is an isomorphism and we define the analytical index map by

$$
\operatorname{Ind}_{a}^{X}:=\left(e_{1}\right)_{*} \circ\left(e_{0}\right)_{*}^{-1}: K_{0}\left(C^{*}\left(T^{\mathrm{S}} X\right)\right) \rightarrow K_{0}(\mathcal{K}) \simeq \mathbb{Z},
$$

or in other words, as the map defined by the Kasparov product with $\partial$. 
Remarks 4.2. 1. Notice that in the case of a smooth manifold with the usual definition of tangent space and tangent groupoid, this definition leads to the classical definition of the analytical index map ([16] II.5).

2. One can associate to a Lie groupoid a different analytical map. More precisely, when $G \rightrightarrows M$ is smooth, one can consider the adiabatic groupoid which is a deformation groupoid of $G$ on its Lie algebroid $\mathcal{A} G$ [4]:

$$
\left.\left.\mathcal{G}^{t}:=\mathcal{A} G \times\{0\} \sqcup G \times\right] 0,1\right] \rightrightarrows M \times[0,1] .
$$

Under some asumption $\mathcal{G}^{t}$ defines a $K K$-element in $K K\left(C^{*}(\mathcal{A} G), C^{*}(G)\right)$ and thus a map from $K_{0}\left(C^{*}(\mathcal{A} G)\right)$ to $K_{0}\left(C^{*}(G)\right)$.

Now, suppose for simplicity that $X$ has only one singularity. In this situation, we will often use the following notation $X_{+}:=X^{\circ} \backslash O_{X}$. The S-tangent space of $X$ is then

$$
T^{\mathrm{S}} X=O_{X} \times O_{X} \sqcup T X_{+} \rightrightarrows X^{\circ}
$$

where $O_{X} \times O_{X} \rightrightarrows O_{X}$ is the pair groupoid and $T X_{+}:=\left.T X^{\circ}\right|_{X_{+}}$. Then we have the following exact sequence of $C^{*}$-algebras:

$$
0 \rightarrow \underbrace{C^{*}\left(O_{X} \times O_{X}\right)}_{=\mathcal{K}\left(L^{2}\left(O_{X}\right)\right)} \stackrel{i}{\longrightarrow} C^{*}\left(T^{\mathrm{S}} X\right) \stackrel{r_{+}}{\longrightarrow} C^{*}\left(T X_{+}\right) \rightarrow 0,
$$

where $i$ is the inclusion morphism and $r_{+}$comes from the restriction of functions. Moreover up to a Fourier transform $C^{*}\left(T X_{+}\right) \simeq C_{0}\left(T^{*} X_{+}\right)$.

Proposition 4.3. The exact sequence (4.2) induces the short exact sequence

$$
0 \rightarrow K_{0}(\mathcal{K}) \simeq \mathbb{Z} \stackrel{i_{*}}{\longrightarrow} K_{0}\left(C^{*}\left(T^{\mathrm{S}} X\right)\right) \stackrel{\left(r_{+}\right)_{*}}{\longrightarrow} K_{0}\left(C_{0}\left(T^{*} X_{+}\right)\right) \rightarrow 0 .
$$

Moreover $\operatorname{Ind}_{a}^{X} \circ i_{*}=I d_{\mathbb{Z}}$, thus

$$
\left(\operatorname{Ind}_{a}^{X},\left(r_{+}\right)_{*}\right): K_{0}\left(C^{*}\left(T^{S} X\right)\right) \rightarrow \mathbb{Z} \oplus K_{0}\left(C_{0}\left(T^{*} X_{+}\right)\right)
$$

is an isomorphism.

Proof. In order to prove the first statement, let us first consider the six terms exact sequence associated to the exact sequence of $C^{*}$-algebra of 4.2 Then recall that $K_{1}(\mathcal{K})=0$. It remains to show that $i_{*}$ is injective. This point is a consequence of the second statement which is proved here'after.

Let $\mathcal{G}_{X}^{t} \rightrightarrows X^{\circ} \times[0,1]$ be a tangent groupoid for $X$. Its restriction $\left.\mathcal{G}_{X}^{t}\right|_{O_{X} \times[0,1]}$ to $O_{X} \times[0,1]$ is isomorphic to the groupoid $\left(O_{X} \times O_{X}\right) \times[0,1] \rightrightarrows O_{X} \times[0,1]$, the pair groupoid of $O_{X}$ parametrized by $[0,1]$. The inclusion of $C_{0}\left(\left.\mathcal{G}_{X}^{t}\right|_{O_{X} \times[0,1]}\right)$ in $C_{0}\left(\mathcal{G}_{X}^{t}\right)$ induces a morphism of $C^{*}$-algebras

$$
i^{t}: C^{*}\left(\left.\mathcal{G}_{X}^{t}\right|_{O_{X} \times[0,1]}\right) \simeq \mathcal{K}\left(L^{2}\left(O_{X}\right)\right) \otimes C([0,1]) \rightarrow C^{*}\left(\mathcal{G}_{X}^{t}\right) .
$$

Moreover, we have the following commutative diagram of $C^{*}$-algebras morphisms:

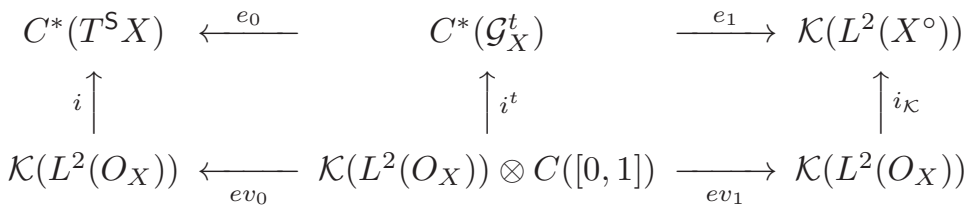


where $i_{\mathcal{K}}$ is the isomorphism induced by the inclusion of the pair groupoid of $O_{X}$ in the pair groupoid of $X^{\circ}$, and $e v_{0}, e v_{1}$ are the evaluations map at 0 and 1 . The $K K$-element $\left[e v_{0}\right]$ is invertible and

$$
\left[e v_{0}\right]^{-1} \otimes\left[e v_{1}\right]=1 \in K K\left(\mathcal{K}\left(L^{2}\left(O_{X}\right)\right), \mathcal{K}\left(L^{2}\left(O_{X}\right)\right)\right) .
$$

Moreover $\cdot \otimes\left[i_{\mathcal{K}}\right]$ induces an isomorphism from $K K\left(\mathbb{C}, \mathcal{K}\left(L^{2}\left(O_{X}\right)\right)\right) \simeq \mathbb{Z}$ onto $K K\left(\mathbb{C}, \mathcal{K}\left(L^{2}\left(X^{\circ}\right)\right)\right) \simeq \mathbb{Z}$. Thus $[i] \otimes \tilde{\partial}=\left[i_{\mathcal{K}}\right]$, which proves that $\operatorname{Ind}_{a}^{X} \circ i_{*}=\operatorname{Id}_{\mathbb{Z}}$ and ensures that $i_{*}$ is injective.

\section{The InVERSe Thom MAP}

Let $\left(E, \mathrm{~S}_{E}, \mathcal{C}_{E}\right)$ be a conical vector bundle over $\left(X, \mathrm{~S}_{X}, \mathcal{C}_{X}\right)$ and $\pi: E \rightarrow X$ the corresponding projection. We let

$$
\left.\left.\mathcal{H}_{E}:=T^{\mathrm{S}} E \times\{0\} \sqcup^{*} \pi^{*}\left(T^{\mathrm{S}} X\right) \times\right] 0,1\right] \rightrightarrows E^{\circ} \times[0,1]
$$

be the Thom groupoid of $E$, as before. The $C^{*}$-algebra of $\mathcal{H}_{E}$ is nuclear as well as the $C^{*}$-algebra of $T^{\mathrm{S}} E$. Thus $\mathcal{H}_{E}$ defines a $K K$-element:

$$
\partial_{\mathcal{H}_{E}}:=\left[\epsilon_{0}\right]^{-1} \otimes\left[\epsilon_{1}\right] \in K K\left(C^{*}\left(T^{\mathrm{S}} E\right), C^{*}\left(T^{\mathrm{S}} X\right)\right),
$$

where $\epsilon_{1}: C^{*}\left(\mathcal{H}_{E}\right) \rightarrow C^{*}\left(\left.\mathcal{H}_{E}\right|_{E^{\circ} \times\{1\}}\right)=C^{*}\left({ }^{*} \pi^{*}\left(T^{\mathrm{S}} X\right)\right)$ is the evaluation map at 1 and $\epsilon_{0}: C^{*}\left(\mathcal{H}_{E}\right) \rightarrow C^{*}\left(\left.\mathcal{H}_{E}\right|_{E^{\circ} \times\{0\}}\right)=C^{*}\left(T^{\mathrm{S}} E\right)$, the evaluation map at 0 is $K$-invertible.

Definition 5.1. The element $\partial_{\mathcal{H}_{E}} \in K K\left(C^{*}\left(T^{\mathrm{S}} E\right), C^{*}\left(T^{\mathrm{S}} X\right)\right)$ defined by Equation (5.2) will be called the inverse Thom element.

Definition-Proposition 5.2. Let $\mathcal{M}$ be the isomorphism induced by the Morita equivalence between $T^{\mathrm{S}} X$ and ${ }^{*} \pi^{*}\left(T^{\mathrm{S}} X\right)$ and let $\cdot \otimes \partial_{\mathcal{H}_{E}}$ be the right Kasparov product by $\partial_{\mathcal{H}_{E}}$ over $C^{*}\left(T^{\mathrm{S}} E\right)$. Then the following diagram is commutative:

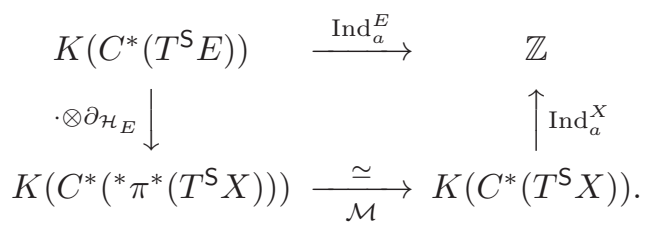

The map $T_{\text {inv }}:=\mathcal{M} \circ\left(\cdot \otimes \partial_{\mathcal{H}_{E}}\right)$ is called the inverse Thom map.

Proof. First consider the deformation groupoid $\mathcal{T}_{E}^{t}$ :

$$
\left.\left.\mathcal{T}_{E}^{t}:=\mathcal{G}_{E}^{t} \times\{0\} \sqcup^{*} \pi_{[0,1]}^{*}\left(\mathcal{G}_{X}^{t}\right) \times\right] 0,1\right] \rightrightarrows E^{\circ} \times[0,1] \times[0,1] .
$$

One can easily see that

$$
\begin{aligned}
\mathcal{T}_{E}^{t} & \left.\left.\left.\left.=T^{\mathrm{S}} E \times\{0\} \times\{0\} \sqcup^{*} \pi^{*}\left(T^{\mathrm{S}} X\right) \times\{0\} \times\right] 0,1\right] \sqcup E^{\circ} \times E^{\circ} \times\right] 0,1\right] \times[0,1] \\
& \left.\left.\simeq \mathcal{H}_{E} \times\{0\} \sqcup\left(E^{\circ} \times E^{\circ} \times[0,1]\right) \times\right] 0,1\right] .
\end{aligned}
$$

The groupoid $\mathcal{T}_{E}^{t}$ is equipped with a smooth structure compatible with the smooth structures of $\left.\left.\mathcal{G}_{E}^{t} \times\{0\},{ }^{*} \pi_{[0,1]}^{*}\left(\mathcal{G}_{X}^{t}\right) \times\right] 0,1\right]$ as well as with the smooth structures of $\mathcal{H}_{E}$ and $\left.\left.\left(E^{\circ} \times E^{\circ} \times[0,1]\right) \times\right] 0,1\right]$. 
We therefore have the following commutative diagram of evaluation morphisms of $C^{*}$-algebras of groupoids:

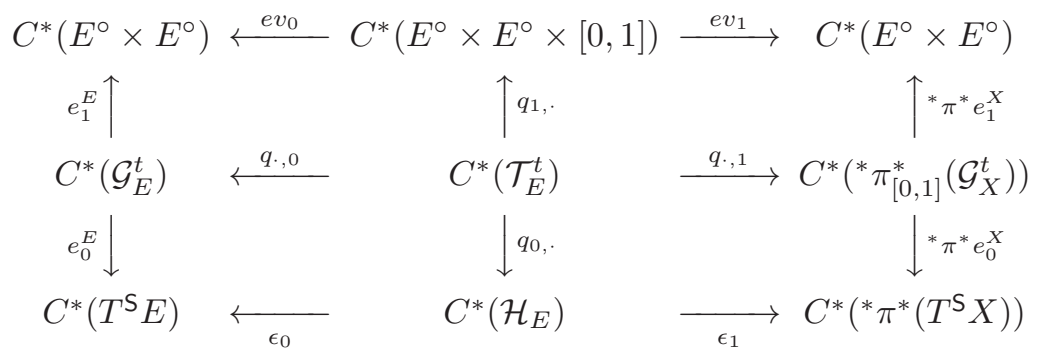

In this diagram, the $K K$-elements $\left[e_{0}^{E}\right],\left[{ }^{*} \pi^{*} e_{0}^{X}\right],[q \cdot, 0],\left[\epsilon_{0}\right],\left[e v_{1}\right]$ and $\left[e v_{0}\right]$ are invertible. Let $\mathcal{M}: K\left(C^{*}\left({ }^{*} \pi^{*}\left(T^{\mathrm{S}} X\right)\right) \rightarrow K\left(C^{*}\left(T^{\mathrm{S}} X\right)\right)\right.$ be the isomorphism induced by the Morita equivalence between ${ }^{*} \pi^{*}\left(T^{\mathrm{S}} X\right)$ and $T^{\mathrm{S}} X$. Also, let $x$ belong to $K\left(C^{*}\left({ }^{*} \pi^{*}\left(T^{\mathrm{S}} X\right)\right)\right)=K K\left(\mathbb{C},{ }^{*} \pi^{*}\left(T^{\mathrm{S}} X\right)\right)$. Then one can easily check the equality

$$
\mathcal{M}(x) \otimes \tilde{\partial}=x \otimes\left[{ }^{*} \pi^{*} e_{0}^{X}\right]^{-1} \otimes\left[{ }^{*} \pi^{*} e_{1}^{X}\right] .
$$

Of course $\left[e v_{0}\right]^{-1} \otimes\left[e v_{1}\right]=1 \in K K\left(C^{*}\left(E^{\circ} \times E^{\circ}\right), C^{*}\left(E^{\circ} \times E^{\circ}\right)\right.$. Thus the previous diagram implies that for any $x \in K\left(C^{*}\left(T^{\mathcal{S}} E\right)\right)=K K\left(\mathbb{C}, C^{*}\left(T^{\mathcal{S}} E\right)\right)$ we have:

$$
\begin{aligned}
\operatorname{Ind}_{a}^{X} \circ T_{i n v}(x) & =x \otimes\left[\epsilon_{0}\right]^{-1} \otimes\left[\epsilon_{1}\right] \otimes\left[{ }^{*} \pi^{*} e_{0}^{X}\right]^{-1} \otimes\left[{ }^{*} \pi^{*} e_{1}^{X}\right] \otimes b \\
& =x \otimes\left[e_{0}^{E}\right]^{-1} \otimes\left[e^{E_{1}}\right] \otimes\left[e v_{0}\right]^{-1} \otimes\left[e v_{1}\right] \otimes b \\
& =\operatorname{Ind}_{a}^{E}(x)
\end{aligned}
$$

\section{INDEX THEOREM}

In this section, we state and prove our main theorem, namely, a topological index theorem for conical pseudomanifolds in the setting of groupoids. We begin with an account of the classical Atiyah-Singer topological index theorem in our groupoid setting.

6.1. A variant of the proof of Atiyah-Singer index theorem for compact manifolds using groupoids. Let $\mathcal{V}$ be the normal bundle of an embedding of a smooth manifold $M$ in some euclidean space. In this subsection, we shall first justify the terminology of "inverse Thom map" we introduced for the map $T_{i n v}$ of Proposition 5.2 by showing that it coincides with the inverse of the classical Thom isomorphism when $E=T \mathcal{V}$ and $X=T M$.

In fact, we will define the Thom isomorphism when $X$ is a locally compact space and $E=N \otimes \mathbb{C}$ is the complexification of a real vector bundle $N \rightarrow X$. As a consequence, we will derive a simple proof of the Atiyah-Singer index theorem for closed smooth manifolds. Our approach has the advantage that it extends to the singular setting.

Let us recall some classical facts 2, 4. If $p: E \rightarrow X$ is a complex vector bundle over a locally compact space $X$, one can define a Thom map

$$
i_{!}: K^{0}(X) \rightarrow K^{0}(E)
$$

which turns to be an isomorphism. This Thom map is defined as follows. Let $x \in K^{0}(X)$ be represented by $\left[\xi_{0} ; \xi_{1} ; \alpha\right]$ where $\xi_{0}, \xi_{1}$ are complex vector bundles over $X$ and $\alpha: \xi_{0} \rightarrow \xi_{1}$ is an isomorphism outside a compact subset of $X$. With no 
lost of generality, one can assume that $\xi_{0}, \xi_{1}$ are hermitian and that $\alpha$ is unitary outside a compact subset of $X$.

Let us consider next the endomorphism of the vector bundle $p^{*}(\Lambda E) \rightarrow E$ given by

$$
(C \omega)(v)=C(v) \omega(v)=\frac{1}{\sqrt{1+\|v\|^{2}}}\left(v \wedge \omega(v)-v^{*}\llcorner\omega(v))\right.
$$

The endomorphism $C \omega$ is selfadjoint, of degree 1 with respect to the $\mathbb{Z}_{2}$-grading $\Lambda_{0}=\Lambda^{\text {even }} E, \Lambda_{1}=\Lambda^{\text {odd }} E$ of the space of exterior forms. Moreover, we have that $(C \omega)^{2} \rightarrow 1$ as $\omega$ approaches infinity in the fibers of $E$. Then, as we shall see in the next Proposition, the Thom morphism $i_{\text {! }}$ of Equation (6.1) can be expressed, in terms of the Kasparov products, as

$$
i_{!}(x):=\left[\xi_{0} \otimes \Lambda_{0} \oplus \xi_{1} \otimes \Lambda_{1} ; \xi_{0} \otimes \Lambda_{1} \oplus \xi_{1} \otimes \Lambda_{0} ; \theta=\left(\begin{array}{cc}
N(1 \otimes C) & M\left(\alpha^{*} \otimes 1\right) \\
M(\alpha \otimes 1) & -N(1 \otimes C)
\end{array}\right)\right]
$$

where $M$ and $N$ are the multiplication operators by the functions $M(v)=\frac{1}{\|v\|^{2}+1}$ and $N=1-M$, respectively.

Proposition 6.1. Let $p: E \longrightarrow X$ be a complex vector bundle over a locally compact base space $X$ and $i_{!}: K^{0}(X) \longrightarrow K^{0}(E)$ the corresponding Thom map. Denote by $T$ the Kasparov element

$$
T:=\left(C_{0}\left(E, p^{*}(\Lambda E)\right), \rho, C\right) \in K K\left(C_{0}(X), C_{0}(E)\right)
$$

where $\rho$ is multiplication by functions. Then $i_{!}(x)=x \otimes T$ for any $x \in K^{0}(X)$.

Proof. The isomorphism $K^{0}(X) \simeq K K\left(\mathbb{C}, C_{0}(X)\right)$ is such that to the triple $\left[\xi_{0} ; \xi_{1} ; \alpha\right]$ there corresponds to the Kasparov module:

$$
x=\left(C_{0}(X, \xi), 1, \widetilde{\alpha}\right), \quad \xi=\xi_{0} \oplus \xi_{1} \text { and } \widetilde{\alpha}=\left(\begin{array}{cc}
0 & \alpha^{*} \\
\alpha & 0
\end{array}\right) .
$$

Similarly, $i_{!}(x)$ corresponds to $(\mathcal{E}, \widetilde{\theta})$ where:

$$
\mathcal{E}=C_{0}(X, \xi) \underset{\rho}{\otimes} C_{0}\left(E, p^{*}(\Lambda E)\right) \simeq C_{0}\left(E, p^{*}(\xi \otimes \Lambda E)\right) \text { and } \widetilde{\theta}=\left(\begin{array}{cc}
0 & \theta^{*} \\
\theta & 0
\end{array}\right) \in \mathcal{L}(\mathcal{E}) \text {. }
$$

We next use the language of 10 55, where the notion of "connection" in the framework of Kasparov's theory was defined. It is easy to check that $M(\widetilde{\alpha} \hat{\otimes} 1)$ is a 0 -connection on $\mathcal{E}$ and $N(1 \hat{\otimes} C)$ is a $C$-connection on $\mathcal{E}$ (the symbol $\hat{\otimes}$ denotes the graded tensor product), which yields that:

$$
\widetilde{\theta}=M(\widetilde{\alpha} \hat{\otimes} 1)+N(1 \hat{\otimes} C)
$$

is a $C$-connection on $\mathcal{E}$. Moreover, for any $f \in C_{0}(X)$, we have

$$
f[\widetilde{\alpha} \hat{\otimes} 1, \widetilde{\theta}] f^{*}=2 M|f|^{2} \widetilde{\alpha}^{2} \hat{\otimes} 1 \geq 0,
$$

which proves that $(\mathcal{E}, \widetilde{\theta})$ represents the Kasparov product of $x$ and $T$.

It is known that $T$ is invertible in $K K$-theory (30, paragraph 5, theorem 8). We now give a description of its inverse via a deformation groupoid when the bundle $E$ is the complexification of a real euclidean bundle $N$. Hence let us assume that $E=N \otimes \mathbb{C}$ or, up to a $\mathbb{C}$-linear vector bundle isomorphism, let us assume that the bundle $E$ is the Withney sum $N \oplus N$ of two copies of some real euclidean vector bundle $p_{N}: N \rightarrow X$ with the complex structure given by $J(v, w)=(-w, v)$, 
$(v, w) \in N \oplus N$. We endow the complex bundle $E$ with the induced hermitian structure. We then define the Thom groupoid as follows:

$$
\mathcal{I}_{N}:=E \times[0,1] \rightrightarrows N \times[0,1]
$$

with structural morphism given by

$$
\begin{aligned}
r(v, w, 0) & =s(v, w, 0)=(v, 0) \\
r(v, w, t) & =(v, t), \\
s(v, w, t) & =(w, t), \quad t>0 \\
(v, w, 0) \cdot\left(v, w^{\prime}, 0\right) & =\left(v, w+w^{\prime}, 0\right) \text { and } \\
(v, w, t) \cdot(w, u, t) & =(v, u, t) \quad t>0 .
\end{aligned}
$$

Thus, for $t=0$, the groupoid structure of $E$ corresponds to the vector bundle structure given by the first projection $E=N \oplus N \rightarrow N$ while for $t>0$ the groupoid structure of $E$ corresponds to the pair groupoid structure in each fiber $E_{x}=N_{x} \times N_{x}$.

The topology of $\mathcal{I}_{N}$ is inherited from the usual tangent groupoid topology, in particular $\mathcal{I}_{N}$ is a Hausdorff topological groupoid that can be viewed as a continuous field of groupoids over $X$ with typical fiber the tangent groupoid of the typical fiber of the vector bundle $N \rightarrow X$. More precisely, the topology of $\mathcal{I}_{N}$ is such that the map $E \times[0,1] \rightarrow \mathcal{I}_{N}$ sending $(u, v, t)$ to $(u, u+t v, t)$ if $t>0$ and equal to identity if $t=0$ is a homeomorphism.

The family of Lebesgue measures on euclidean fibers $N_{x}, x \in X$, gives rise to a continuous Haar system on $\mathcal{T}_{N}$ that allows us to define the $C^{*}$-algebra of $\mathcal{I}_{N}$ as a continuous field of amenable groupoids. Therefore, $\mathcal{I}_{N}$ is amenable. We also get an element of $K K\left(C^{*}(E), C_{0}(X)\right)$, denoted by $T_{i n v}$ and defined as usual by:

$$
T_{\text {inv }}:=\left[e_{0}\right]^{-1} \otimes\left[e_{1}\right] \otimes \mathcal{M} .
$$

Here, as before, the morphism $e_{0}: C^{*}\left(\mathcal{I}_{N}\right) \rightarrow C^{*}\left(\left.\mathcal{I}_{N}\right|_{t=0}\right)=C^{*}(E)$ is the evaluation at 0 , the morphism $e_{1}: C^{*}\left(\mathcal{I}_{N}\right) \rightarrow C^{*}\left(\left.\mathcal{I}_{N}\right|_{t=1}\right)$ is the evaluation at 1 , and $\mathcal{M}$ is the natural Morita equivalence between $C^{*}\left(\left.\mathcal{I}_{N}\right|_{t=1}\right)$ and $C_{0}(X)$. For instance, $\mathcal{M}$ is represented by the Kasparov module $(\mathcal{H}, m, 0)$ where $\mathcal{H}$ is the continuous field over $X$ of Hilbert spaces $\mathcal{H}_{x}=L^{2}\left(N_{x}\right), x \in X$ and $m$ is the action of $C^{*}\left(\left.\mathcal{I}_{N}\right|_{t=1}\right)=$ $C^{*}(N \underset{X}{\times} N)$ by compact operators on $\mathcal{H}$.

We denote $T_{0}=\left(\mathcal{E}_{0}, \rho_{0}, F_{0}\right) \in K K\left(C_{0}(X), C^{*}(E)\right)$ the element corresponding to the Thom element $T$ of proposition 6.1 trough the isomorphism $C_{0}(E) \simeq C^{*}(E)$. This isomorphism is given by the Fourier transform applied to the second factor in $E=N \oplus N$ provided with the groupoid structure of $\left.\mathcal{I}_{N}\right|_{t=0}$. The $C^{*}(E)$-Hilbert module $\mathcal{E}_{0}=C^{*}(E, \Lambda E)$ is the natural completion of $C_{c}\left(E, p^{*}(\Lambda E)\right.$ ) ( $p$ is the bundle map $E \rightarrow X)$. The representation $\rho_{0}$ of $C_{0}(X)$ and the endomorphism $F_{0}$ of $\mathcal{E}_{0}$ are given by

$$
\begin{gathered}
\rho_{0}(f) \omega(v, w)=f(x) \omega(v, w), \\
F_{0} \omega(v, w)=\int_{\left(w^{\prime}, \xi\right) \in N_{x} \times N_{x}^{*}} e^{i\left(w-w^{\prime}\right) \cdot \xi} C(v+i \xi) \omega\left(v, w^{\prime}\right) d w^{\prime} d \xi .
\end{gathered}
$$

In the above formulas, $f \in C_{0}(X), \omega \in C_{c}\left(E, p^{*}(\Lambda E)\right)$ and $(v, w) \in E_{x}$. We can therefore state the following result.

Theorem 6.2. The elements $T_{i n v}$ and $T_{0}$ are inverses to each other in $K K$-theory. 
Proof. We know (30], paragraph 5, theorem 8) that $T$, hence $T_{0}$, is invertible so it is enough to check that $T_{0} \otimes T_{i n v}=1 \in K K\left(C_{0}(X), C_{0}(X)\right)$.

Since $T_{i n v}:=\left[e_{0}\right]^{-1} \otimes\left[e_{1}\right] \otimes \mathcal{M}$ where $e_{t}$ are restriction morphisms at $t=0,1$ in the groupoid $\mathcal{I}_{N}$ we first compute $\widetilde{T}=T_{0} \otimes\left[e_{0}\right]^{-1}$, that is, we look for $\widetilde{T}=$ $(\mathcal{E}, \rho, F) \in K K\left(C_{0}(X), C^{*}\left(\mathcal{I}_{N}\right)\right)$ such that

$$
\left(e_{0}\right)_{*}(\widetilde{T})=\left(\mathcal{E} \otimes e_{0} C^{*}(E), \rho, F \otimes 1\right)=T_{0}
$$

Let $\mathcal{E}=C^{*}\left(\mathcal{I}_{N}, \Lambda E\right)$ be the $C^{*}\left(\mathcal{I}_{N}\right)$-Hilbert module completion of $C_{c}\left(\mathcal{I}_{N},\left(r^{\prime}\right)^{*} \Lambda E\right)$, where $r^{\prime}=p \circ \operatorname{pr}_{1} \circ r: \mathcal{I}_{N} \rightarrow X$. Let us define a representation $\rho$ of $C_{0}(X)$ on $\mathcal{E}$ by

$$
\rho(f) \omega(v, w, t)=f(p(v)) \omega(v, w, t) \quad \text { for all } f \in C_{0}(X), \omega \in \mathcal{E},(v, w, t) \in \mathcal{I}_{N}
$$

Let $F$ be the endomorphism of $\mathcal{E}$ densely defined on $C_{c}\left(\mathcal{I}_{N},\left(r^{\prime}\right)^{*} \Lambda E\right)$ by

$$
F \omega(v, w, t)=\int_{\left(v^{\prime}, \xi\right) \in N_{x} \times N_{x}^{*}} e^{i\left(\frac{v-v^{\prime}}{t}\right) \cdot \xi} C(v+i \xi) \omega\left(v^{\prime}, w, t\right) \frac{d v^{\prime}}{t^{n}} d \xi,
$$

if $t>0$ and by $F \omega(v, w, 0)=F_{0} \omega(v, w, 0)$ if $t=0$. The integer $n$ above is the rank of the bundle $N \rightarrow X$. One can check that the triple $(\mathcal{E}, \rho, F)$ is a Kasparov $\left(C_{0}(X), C^{*}\left(\mathcal{I}_{N}\right)\right)$-module and that under the obvious isomorphism

$$
q \mathcal{E} \underset{e_{0}}{\otimes} C^{*}(E) \simeq \mathcal{E}_{0},
$$

$\rho$ coincides with $\rho_{0}$ while $F \otimes 1$ coincides with $F_{0}$.

Next, we evaluate $\widetilde{T}$ at $t=1$ and $T_{1}:=\left(e_{1}\right)_{*}(\widetilde{T}) \in K K\left(C_{0}(X), C^{*}(N \underset{X}{\times} N)\right)$ is represented by $\left(\mathcal{E}_{1}, \rho_{1}, F_{1}\right)$ where $\mathcal{E}_{1}=C^{*}(N \underset{X}{\times} N, \Lambda E)$ is the $C^{*}(N \underset{X}{\times} N)$-Hilbert module completion of $C_{c}\left(\left.\mathcal{I}_{N}\right|_{t=1},(p \circ r)^{*} \Lambda E\right)$ and $\rho_{1}, F_{1}$ are given by the formulas above where $t$ is replaced by 1 .

Now, applying the Morita equivalence $\mathcal{M}$ to $T_{1}$ gives:

$$
\left(\mathcal{E}_{1}, \rho_{1}, F_{1}\right) \otimes(\mathcal{H}, m, 0)=\left(\mathcal{H}_{\Lambda E}, \phi, F_{1}\right),
$$

where $\mathcal{H}_{\Lambda E}=\left(L^{2}\left(N_{x}, \Lambda E_{x}\right)\right)_{x \in X}, \phi$ is the obvious action of $C_{0}(X)$ on $\mathcal{H}_{\Lambda E}$ and $F_{1}$ is the same operator as above identified with a continuous family of Fredholm operators acting on $L^{2}\left(N_{x}, \Lambda E_{x}\right)$ :

$$
F_{1} \omega(x, v)=\int_{\left(v^{\prime}, \xi\right) \in N_{x} \times N_{x}^{*}} e^{i\left(v-v^{\prime}\right) \cdot \xi} C(v+i \xi) \omega\left(x, v^{\prime}\right) d v^{\prime} d \xi .
$$

By (18] lemma 2.4) we know that $\left(\mathcal{H}_{\Lambda E}, \phi, F_{1}\right)$ represents 1 in $K K\left(C_{0}(X), C_{0}(X)\right)$ (the key point is again that the equivariant $O_{n}$-index of $F_{1}$ restricted to even forms is 1 , see also [28]) and the theorem is proved.

Now let us consider the vector bundle $p: T \mathcal{V} \longrightarrow T M$, where $M$ is a compact manifold embedded in some $\mathbb{R}^{N}$ and $\mathcal{V}$ is the normal bundle of the embedding. We let $q: T M \rightarrow M$ be the canonical projection and to simplify notations, we denote again by $p$ the bundle map $\mathcal{V} \rightarrow M$ and by $\mathcal{V}$ the pull-back of $\mathcal{V}$ to $T M$ via $q$.

Using the identifications $T_{x} M \oplus \mathcal{V}_{x} \simeq T_{(x, v)} \mathcal{V}$ for all $x \in M$ and $v \in \mathcal{V}_{x}$, we get the isomorphism of vector bundles over $T M$ :

$$
q^{*}(\mathcal{V} \oplus \mathcal{V}) \ni(x, X, v, w) \longmapsto(x, v ; X+w) \in T \mathcal{V} .
$$


It follows that $T \mathcal{V}$ inherits a complex structure from $\mathcal{V} \oplus \mathcal{V} \simeq \mathcal{V} \otimes \mathbb{C}$ and we take the Atiyah-Singer convention: via the above isomorphism, the first parameter is real and the second is imaginary.

The previous construction leads to the groupoid $\mathcal{I}_{\mathcal{V}}$ giving the inverse of the Thom isomorphism. Actually, we slighty modify to retain the natural groupoid structure carried by the base space $T M$ of the vector bundle $T \mathcal{V}$ (it is important in the purpose of extending the Thom isomorphism to the singular setting). Thus, we set:

$$
\left.\left.\mathcal{H}_{\mathcal{V}}=T \mathcal{V} \times\{0\} \sqcup^{*} p^{*}(T M) \times\right] 0,1\right] \rightrightarrows \mathcal{V} \times[0,1]
$$

This is the Thom groupoid defined in the section 3.2 The groupoids $\mathcal{I}_{\mathcal{V}}$ and $\mathcal{H}_{\mathcal{V}}$ are not isomorphic, but a Fourier transform in the fibers of $T M$ provides an isomorphism of their $C^{*}$-algebras: $C^{*}\left(\mathcal{I}_{\mathcal{V}}\right) \simeq C^{*}\left(\mathcal{H}_{\mathcal{V}}\right)$. Moreover, this isomorphism is compatible with the restrictions morphisms and we can rewrite the theorem (6.2):

Corollary 6.3. Let $\partial_{\mathcal{H}_{\mathcal{V}}}=\left[\epsilon_{0}\right]^{-1} \otimes\left[\epsilon_{1}\right]$ be the $K K$-element associated with the deformation groupoid $\mathcal{H}_{\mathcal{V}}$ and let $\mathcal{M}$ be the natural Morita equivalence between $C^{*}\left({ }^{*} p^{*}(T M)\right)$ and $C^{*}(T M)$. Then $T_{i n v}=\partial_{\mathcal{H} \mathcal{V}} \otimes \mathcal{M} \in K K\left(C^{*}(T \mathcal{V}), C^{*}(T M)\right)$ gives the inverse of the Thom isomorphism $T \in K K\left(C_{0}\left(T^{*} M\right), C_{0}\left(T^{*} \mathcal{V}\right)\right.$ trough the isomorphisms $C_{0}\left(T^{*} M\right) \simeq C^{*}(T M)$ and $C_{0}\left(T^{*} \mathcal{V}\right) \simeq C^{*}(T \mathcal{V})$.

Remarks 6.4. 1) Let us assume that $M$ is a point and $\mathcal{V}=\mathbb{R}^{N}$. The groupoid $\mathcal{H}_{\mathcal{V}}$ is equal in that case to the tangent groupoid of the manifold $\mathbb{R}^{N}$ and the associated $K K$-element $\partial_{\mathcal{H}} \otimes \mathcal{M}$ gives the Bott periodicity between the point and $\mathbb{R}^{2 N}$.

2) Let $M_{+}$be a compact manifold with boundary and $M$ the manifold without boundary obtained by doubling $M_{+}$. Keeping the notations above, let $\mathcal{V}_{+}$be the restriction of $\mathcal{V}$ to $M_{+}$. All the previous constructions applied to $M$ restrict to $M_{+}$ and give the inverse $T_{i n v}^{+}$of the Thom element $T^{+} \in K K\left(C_{0}\left(T^{*} M_{+}\right), C_{0}\left(T^{*} \mathcal{V}_{+}\right)\right)$.

With this description of the (inverse) Thom isomorphism in hand, the equality between the analytical and topological indices of Atiyah and Singer 2$]$ follows from a commutative diagram. Let us denote by $p_{[0,1]}$ the map $p \times \operatorname{Id}: \mathcal{V} \times[0,1] \rightarrow M \times[0,1]$. We consider the deformation groupoid (cf. example 1. of 3.2)

$\left.\left.\mathcal{T}_{\mathcal{V}}^{t}=\mathcal{G}_{\mathcal{V}}^{t} \times\{0\} \sqcup^{*} p_{[0,1]}^{*}\left(\mathcal{G}_{M}^{t}\right) \simeq \mathcal{H}_{\mathcal{V}} \times\{0\} \sqcup(\mathcal{V} \times \mathcal{V} \times[0,1]) \times\right] 0,1\right] \rightrightarrows \mathcal{V} \times[0,1] \times[0,1]$

We use the obvious notation for restriction morphisms (cf. proof of definitionproposition [5.2) and $\mathcal{M}$ for the various (but always obvious) Morita equivalence 
maps. To shorten the diagram, we set $K(G):=K_{0}\left(C^{*}(G)\right)$ for all the (amenable) groupoids met below. We have:

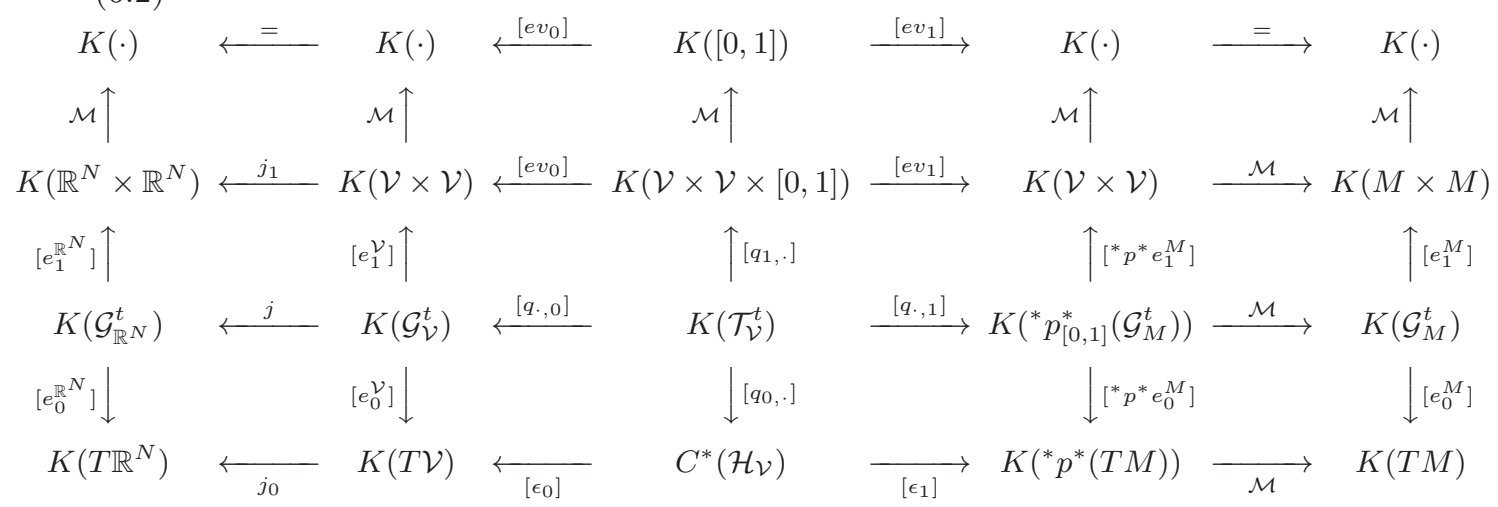

The commutativity of this diagram is obvious. From the previous remark we deduce that the map $K\left(T \mathbb{R}^{N}\right) \rightarrow \mathbb{Z}$ associated with $\left[e_{0}^{\mathbb{R}^{N}}\right]^{-1} \otimes\left[e_{1}^{\mathbb{R}^{N}}\right] \otimes \mathcal{M}$ on the left column is equal to the Bott periodicity isomorphism $\beta$. Thanks to the corollary [6.3), the map $K(T M) \rightarrow K(T \mathcal{V})$ associated with $\mathcal{M}^{-1} \otimes \partial_{H_{\mathcal{V}}}^{-1}=T_{0}$ on the bottom line is equal to the Thom isomorphism, while $j_{0}$ is the usual excision map resulting from the identification of $\mathcal{V}$ with an open subset of $\mathbb{R}^{N}$. It follows that composing the bottom line with the left column produces the map:

$$
\beta \circ j_{0} \circ T_{0}: K(T M) \rightarrow \mathbb{Z}
$$

which is exaclty the Atiyah-Singer's definition of the topological index map. We already know that the map $K(T M) \rightarrow \mathbb{Z}$ associated with $\left[e_{0}^{M}\right]^{-1} \otimes\left[e_{1}^{M}\right] \otimes \mathcal{M}$ on the right column is the analytical index map. Finally, the commutativity of the diagram and the fact that the map associated with $\left[e v_{0}\right]^{-1} \otimes\left[e v_{1}\right]$ on the top line is identity, completes our proof of the Atiyah-Singer index theorem.

Another proof of the usual Atiyah-Singer index theorem in the framework of deformation groupoids can be found in [40].

6.2. An index theorem for conical pseudomanifolds. We define for a conical manifold a topological index and prove the equality between the topological and analytical indices. Both indices are straight generalisations of the ones used in the Atiyah-Singer index theorem: indeed, if we apply our constructions to a smooth manifold and its tangent space, we find exaclty the classical topological and analytical indices. Thus, the egality of indices we proove can be presented as the index theorem for conical manifolds. Moreover, the $K$-theory of $T^{\mathrm{S}} X$ is exhausted by elliptic symbols associated with pseudo-differential operators in the $b$-calculus 33 . and the analytical index can be interpreted via the Poincaré duality [21], as their Fredholm index.

Let $X$ be a conical pseudomanifold embedded in $\mathbb{R}^{N}$ for some $N$ and let $\mathcal{W}$ be a tubular neighborhood of this embedding as in 1 We assume for simplicity that $X$ has only one singularity. We denote by

$$
\left.\left.\mathcal{H}_{\mathcal{W}}=T^{\mathrm{S}} \mathcal{W} \times\{0\} \sqcup^{*} \pi^{*}\left(T^{\mathrm{S}} X\right) \times\right] 0,1\right] \rightrightarrows \mathcal{W}^{\circ} \times[0,1]
$$


the Thom groupoid associated with $\pi: \mathcal{W} \rightarrow X$ and by

$$
\left.\left.\mathcal{H}_{+}=T \mathcal{W}_{+} \times\{0\} \sqcup^{*} \pi_{+}^{*}\left(T X_{+}\right) \times\right] 0,1\right] \rightrightarrows \mathcal{W}_{+} \times[0,1]
$$

the Thom groupoid associated with $\pi_{+}: \mathcal{W}_{+} \rightarrow X_{+}$. Recall that $\mathcal{W}_{+}=\mathcal{W} \backslash O_{\mathcal{W}}=$ $\{(z, V) \in \mathcal{W} \mid \rho(z) \geq 1\}$ and $X_{+}=X \backslash O_{X}=\{z \in X \mid \rho(z) \geq 1\}$, where $\rho$ is in both case the defining function. We denote by $T_{i n v}$ and $T_{i n v}^{+}$the respective inverse-Thom elements. Recall (cf. prop. 4.3) that we have the two following short exact sequences comming from inclusion and restriction morphisms:

$$
\begin{aligned}
& 0 \longrightarrow K\left(\mathcal{K}\left(L^{2}\left(O_{\mathcal{W}}\right)\right)\right) \stackrel{i \mathcal{V}_{*}}{\longrightarrow} K\left(C^{*}\left(T^{\mathrm{S}} \mathcal{W}\right)\right) \stackrel{r \mathcal{W}_{*}}{\longrightarrow} K\left(C^{*}\left(T \mathcal{W}_{+}\right)\right) \longrightarrow 0 \\
& 0 \longrightarrow K\left(\mathcal{K}\left(L^{2}\left(O_{X}\right)\right)\right) \longrightarrow K\left(C^{*}\left(T^{\mathrm{S}} X\right)\right) \longrightarrow 0
\end{aligned}
$$

Definition-Proposition 6.5. The following diagram commutes:

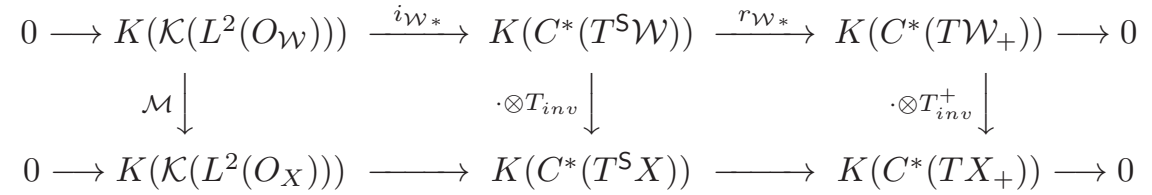

where $\mathcal{M}$ is the natural Morita equivalence map. In particular, the map:

$$
\cdot \otimes T_{i n v}: K\left(C^{*}\left(T^{\mathrm{S}} \mathcal{W}\right)\right) \longrightarrow K\left(C^{*}\left(T^{\mathrm{S}} X\right)\right)
$$

is an isomorphism. Its inverse is denoted by $T$ and called the Thom isomorphism.

Proof. Let us note again by $\pi$ the (smooth) vector bundle map $\mathcal{W}^{\circ} \rightarrow X^{\circ}$ and consider the following diagram:

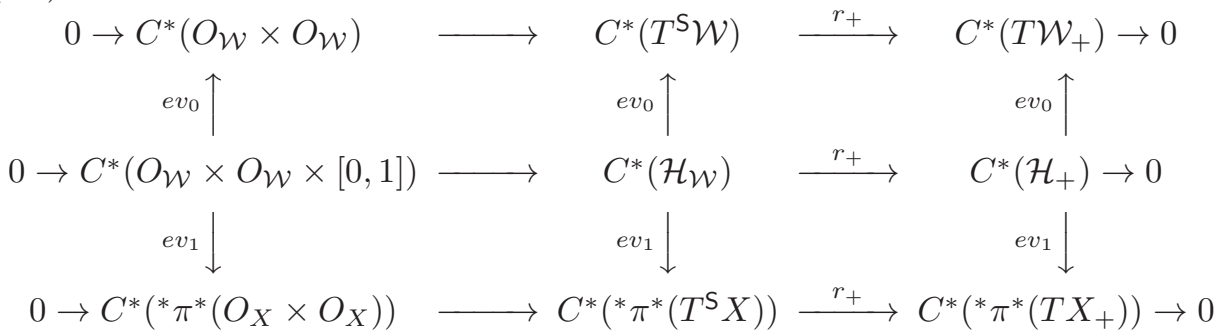

where the (Lie) groupoid isomorphism * $\pi^{*}\left(O_{X} \times O_{X}\right) \simeq O_{\mathcal{W}} \times O_{\mathcal{W}}$ has been used. Applying the $K$ functor and Morita equivalence maps to the bottom line to get rid of the pull back ${ }^{*} \pi^{*}$ and using the fact that the long exact sequences in $K$-theory associated to the top and bottom lines split in short exact sequences, give the diagram (6.3). Since $\mathcal{M}$ and $T_{i n v}^{+}$are isomorphisms, the same is true for $T_{i n v}$.

Remarks 6.6. 1) When $X$ has several singular points, the invertibility of . $\otimes$ $T_{\text {inv }}$ remains true. To check it, consider, with notations of remark 3.3. the exact sequence:

$$
0 \rightarrow \bigoplus_{s \in \mathrm{S}} \mathcal{K}\left(L^{2}\left(O_{s}\right)\right) \longrightarrow C^{*}\left(T^{\mathrm{S}} X\right) \longrightarrow C^{*}\left(\left.T X^{\circ}\right|_{X^{\circ} \backslash O}\right) \rightarrow 0
$$

as well as the corresponding exact sequence for $T^{\mathrm{S}} \mathcal{W}$. We then get a diagram like (6.3) except that long exact sequences in $K$-theory do not split. One can check the commutativity of this diagram, hence the five lemma allows us to complete the argument. 
2) The Thom map we define extends the usual one: this is exactly what is said by the commutativity of the diagram (6.3).

Let us recall that we started with an embedding of $X$ into $\left(\mathbb{R}^{N}\right)^{c}=c \mathbb{R}^{N-1}$. The S-tangent space $T^{S} \mathcal{W}$ of $\mathcal{W}$ is obviously isomorphic to an open subgroupoid of the S-tangent space $T^{S}\left(\mathbb{R}^{N}\right)^{c}$. Thus we get an excision homomorphism:

$$
j: C^{*}\left(T^{\mathrm{S}} \mathcal{W}\right) \longrightarrow C^{*}\left(T^{\mathrm{S}}\left(\mathbb{R}^{N}\right)^{c}\right) .
$$

There is a natural identification of the $K$-theory group $K\left(T^{S}\left(\mathbb{R}^{N}\right)^{c}\right)$ with $\mathbb{Z}$, analog to the one given by Bott periodicity in the case of $T \mathbb{R}^{N}=\mathbb{R}^{2 N}$ coming from its tangent groupoid (cf. remark 6.4):

$$
\begin{gathered}
\partial_{\left(\mathbb{R}^{N}\right)^{c}}=\left[e_{0}\right]^{-1} \otimes\left[e_{1}\right] \otimes \mathcal{M}: K\left(T^{S}\left(\mathbb{R}^{N}\right)^{c}\right) \longrightarrow \mathbb{Z} \\
K\left(C^{*}\left(T^{S}\left(\mathbb{R}^{N}\right)^{c}\right)\right) \stackrel{\left[e_{0}\right]}{\longleftarrow} K\left(C ^ { * } \left(\mathcal{G}_{\left(\mathbb{R}^{N}\right)^{c}}^{t} \stackrel{\left[e_{1}\right]}{\longrightarrow} K\left(\mathcal{K}\left(L^{2}\left(\mathbb{R}^{N}\right)\right)\right) \stackrel{\mathcal{M}}{\rightarrow} K(\cdot) \simeq \mathbb{Z}\right.\right.
\end{gathered}
$$

We are now in position to extend the Atiyah-Singer topological index to conical pseudomanifolds:

Definition 6.7. The topological index of the conical pseudomanifold $X$ is defined by

$$
\operatorname{Ind}_{t}^{X}=\partial_{\left(\mathbb{R}^{N}\right)^{c}} \circ[j] \circ T
$$

Moreover, we obtain the following extension of the Atiyah-Singer Index theorem

Theorem 6.8. If $X$ is a pseudomanifold with conical singularities then

$$
\operatorname{Ind}_{a}^{X}=\operatorname{Ind}_{t}^{X}
$$

Proof. The proof is similar to our proof of the Atiyah-Singer index theorem. Indeed, let us write down the analog of the diagram (6.2) for the singular manifold $X$ :

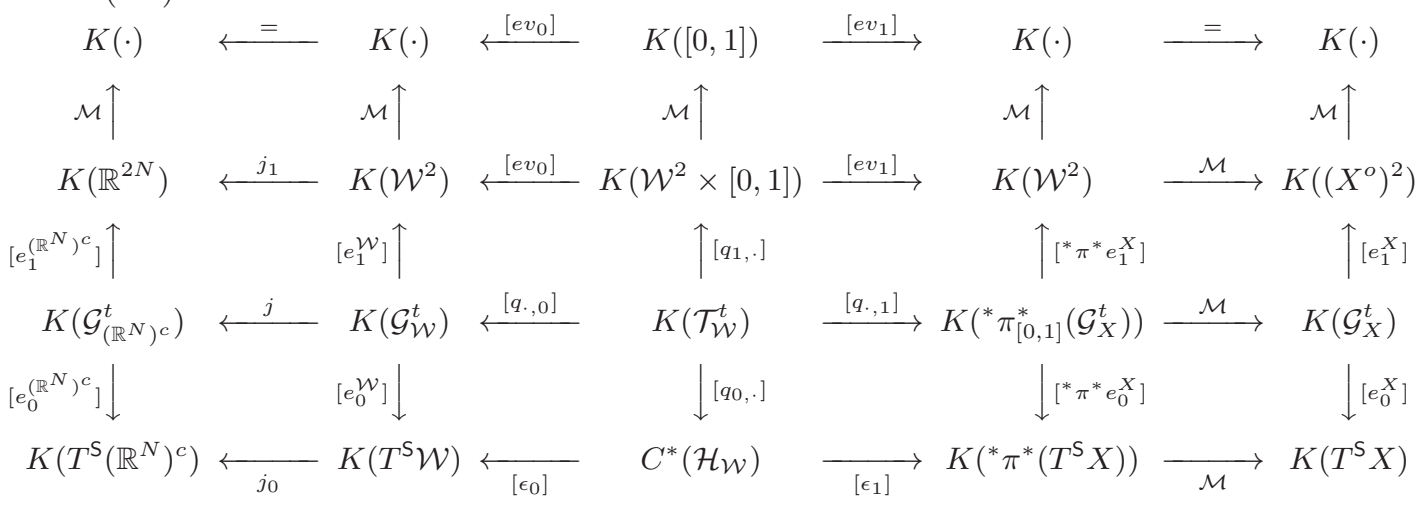

This diagram involves various deformation groupoids associated to $X$ and its embedding into $\left(\mathbb{R}^{N}\right)^{c}$. The commutativity is obvious since everything comes from morphisms of algebras or from explicit Morita equivalences. As before, the convention $K(G)=K_{0}\left(C^{*}(G)\right)$ is used to shorten the diagram and intuitive notations are chosen to name the various restriction morphisms. Starting from the bottom right corner and following the right column gives the analytical index map. Starting from the bottom right corner and following the bottom line and next the left column gives the topological index map. 
A natural question arising with this index map is what are the Fredholm operators whose index is computed?

The answer to this question, briefly discussed in the introduction, lies in the interpretation of the Poincare duality:

$$
K_{0}(X) \stackrel{\Sigma_{X}}{\longrightarrow} K^{0}\left(T^{\mathcal{S}} X\right)
$$

as a symbol map. This is done in full details in [33 and is summarized below.

In the sequel $\Psi_{b}^{*}$ denotes the algebra of the small $b$-calculus for the manifold $X^{o}$. Every fully elliptic $b$-operator of order 0 provides a $K$-homology class of $X$ in a obvious way, and, as a consequence of our Poincare duality, the converse is true. We shall say that a $b$-pseudodifferential operator $P$ is full elliptic if its principal symbol is invertible outside the zero section (interior ellipticity) and its indicial family $\hat{A}(\tau)$ is invertible for all the real values of the parameter $\tau$. One is more comfortable to relate these fully elliptic operators to our non commutative tangent space when dealing with a slightly different, but $K K$-equivalent definition of the tangent space:

$$
\left.T^{q} X=T\right] 0,1\left[\times L \times L \sqcup T X_{+} \rightrightarrows X^{o}\right.
$$

This groupoid is provided with a smooth structure in the same way as $T^{\mathcal{S}} X$ and an obvious deformation between them appears to be a $K K$-equivalence. Trough this $K K$-equivalence we can bring all our constructions to $T^{q} X$.

Let us now turn to the notion of symbols: the algebra of symbols $S^{*}(X)$ is defined as a particular subspace $\mathcal{S}$ of the space of pseudodifferential operators on $T^{q} X$. We require an appropriate behavior of Schwartz kernels of the operators in $\mathcal{S}$, including a smoothness of these kernels up to $h=0$. This definition of symbols may look strange at first glance, but recall that this is analogous to the way symbols are defined on a smooth manifold. Namely, up to a Fourier transform, the quantization of symbols assigns to a suitable function on the cotangent space a family of convolution operators on the tangent space. The notion of ellipticity results from the expectation that elliptic symbols should provide $K$-theory classes of the tangent space $T^{q} X$. Note that 0-order symbols are bounded multipliers of $C^{*}\left(T^{q} X\right)$, but $(-1)$-order symbols are not in $C^{*}\left(T^{q} X\right)$, actually :

$$
S^{-1}(X) \cap C^{*}\left(T^{q} X\right)=S_{0}^{-1}(X)
$$

where the subscript 0 on the right hand side means that we consider symbols vanishing at $h=0$. Thus we make the following definition : a symbol $a \in S^{m}(X)$ is elliptic if there exists $b \in S^{m-1}(X)$ such that $a b-1$ and $b a-1$ belong to $S_{0}^{-1}(X)$. Now, to an ellitpic symbol $a \in S^{0}(X)$ corresponds canonically a $K$-theory class $[a] \in K^{0}\left(T^{q} X\right)$. Moreover, using again an appropriate deformation of groupoids we get:

Theorem 6.9. 33] There exist a linear map :

$$
\Psi_{b}^{*} \stackrel{\sigma}{\longrightarrow} S^{*}(X)
$$

such that :

(1) $\sigma$ is an algebra homomorphism up to lower order terms,

(2) $P \in \Psi_{b}^{*}$ is fully elliptic if and only if $\sigma(P)$ is elliptic,

(3) Given a fully elliptic operator $P \in \Psi_{b}^{0}$, the Poincare dual of $[P] \in K_{0}(X)$ is given by $[\sigma(P)] \in K^{0}\left(T^{q} X\right)$. 
See also [32]. Allowing vector bundles over $X^{o}$ and symbols acting between vector bundles, one can proove that elliptic symbols generate the $K$-theory of the tangent space $T^{q} X$ and the theorem above can be easely extended to this case, leading to the complete explicitation of the Poincare duality. In other words, trough the $K K$-equivalence between $T^{q} X$ and $T^{\mathcal{S}} X$ we have proved:

$$
\operatorname{Ind}^{X}(\sigma(P))=\operatorname{Ind} P
$$

where $P \in \Psi_{b}^{0}$ is fully elliptic and Ind $P$ is its index as a Fredholm operator on a natural $L^{2}$ space it acts on.

\section{REFERENCES}

[1] B. Ammann, R. Lauter, and V. Nistor. Pseudodifferntial operators on manifolds with a Lie structure at infinity. Preprint, to appear in Ann. of Math., December 2002.

[2] M. Atiyah and I. Singer. The index of elliptic operators I, III. Annals of Math., 87:484530,546-604, 1968.

[3] M. F. Atiyah, V. K. Patodi, and I. M. Singer. Spectral asymmetry and Riemannian geometry. I. Math. Proc. Cambridge Philos. Soc., 77:43-69, 1975.

[4] M.F. Atiyah. K-theory. Advanced Book Classics. Addison-Wesley Publishing Company Advanced Book Program, Redwood City, CA, 1989. Notes by D. W. Anderson.

[5] C. Băcuţă, V. Nistor, and L. Zikatanov. Improving the rate of convergence of 'high order finite elements' on polygons and domains with cusps. Numer. Math., 100(2):165-184, 2005.

[6] M.-T. Benameur and V. Nistor. Homology of algebras of families of pseudodifferential operators. J. Funct. Anal., 205(1):1-36, 2003.

[7] J.-M. Bismut. The Atiyah-Singer theorems: a probabilistic approach. I. The index theorem. J. Funct. Anal., 57(1):56-99, 1984.

[8] J.-M. Bismut. The Atiyah-Singer theorems: a probabilistic approach. II. The Lefschetz fixed point formulas. J. Funct. Anal., 57(3):329-348, 1984.

[9] J.-M. Bismut and D. Freed. The analysis of elliptic families. I. Metrics and connections on determinant bundles. Comm. Math. Phys., 106(1):159-176, 1986.

[10] B. Blackadar. K-theory for operator algebras, volume 5 of Mathematical Sciences Research Institute Publications. Cambridge University Press, Cambridge, second edition, 1998.

[11] J.-P. Brasselet, G. Hector, and Saralegi. Théorème de de Rham pour les variétés stratifiées. Ann. Global Anal. Geom., 9(3):211-243, 1991.

[12] A. Cannas da Silva and A. Weinstein. Geometric Models for Noncommutative Algebras. Berkeley Math. Lecture Notes series, 1999.

[13] C. Carvalho. A topological approach to the cobordism invariance of the index. K-Theory, 2006.

[14] A. Connes. Sur la théorie non commutative de l'intégration. In Algèbres d'opérateurs (Sém., Les Plans-sur-Bex, 1978), volume 725 of Lecture Notes in Math., pages 19-143. Springer, Berlin, 1979.

[15] A. Connes. A survey of foliations and operator algebras. In Operator algebras and applications, Part I (Kingston, Ont., 1980), volume 38 of Proc. Sympos. Pure Math., pages 521-628. Amer. Math. Soc., Providence, R.I., 1982.

[16] A. Connes. Noncommutative Geometry. Academic Press, 1994.

[17] A. Connes and H. Moscovici. The local index formula in noncommutative geometry. Geom. Funct. Anal., 5(2):174-243, 1995.

[18] A. Connes and G. Skandalis. The longitudinal index theorem for foliations. Publ. R.I.M.S. Kyoto Univ., 20:1139-1183, 1984.

[19] M. Crainic and R. Fernandes. Integrability of Lie brackets. Ann. of Math. (2), 157(2):575-620, 2003.

[20] C. Debord. Holonomy groupoids for singular foliations. J. of Diff. Geom., 58:467-500, 2001.

[21] C. Debord and J.-M. Lescure. $K$-duality for pseudomanifolds with isolated singularities. $J$. Funct. Anal., 219(1):109-133, 2005.

[22] B. Fedosov, B.-W. Schulze, and N. Tarkhanov. The index of elliptic operators on manifolds with conical points. Selecta Math. (N.S.), 5(4):467-506, 1999. 
[23] B. V. Fedosov and B.-W. Schulze. On the index of elliptic operators on a cone. In Schrödinger operators, Markov semigroups, wavelet analysis, operator algebras, volume 11 of Math. Top., pages 348-372. Akademie Verlag, Berlin, 1996.

[24] D. Freed and E. Witten. Anomalies in string theory with D-branes. Asian J. Math., 3(4):819$851,1999$.

[25] M. Goresky and R. MacPherson. Intersection homology theory. Topology, 19:135-162, 1980.

[26] A. Gorokhovsky and J. Lott. Local index theory over étale groupoids. J. Reine Angew. Math., 560:151-198, 2003.

[27] M. Hilsum and G. Skandalis. Morphismes $K$-orientés d'espaces de feuilles et fonctorialité en théorie de Kasparov. Ann. Sci. Ecole Norm. Sup., 20 (4):325-390, 1987.

[28] L. Hörmander. On the index of pseudodifferential operators. In Elliptische Differentialgleichungen, Band II, pages 127-146. Schriftenreihe Inst. Math. Deutsch. Akad. Wissensch. Berlin, Reihe A, Heft 8. Akademie-Verlag, Berlin, 1971.

[29] B. Hughes and Sh. Weinberger. Surgery and stratified spaces. In Surveys on surgery theory, Vol. 2, volume 149 of Ann. of Math. Stud., pages 319-352. Princeton Univ. Press, Princeton, NJ, 2001.

[30] G.G. Kasparov. The operator K-functor and extensions of $C^{*}$-algebras. Izv. Akad. Nauk SSSR, Ser. Math., 44:571-636, 1980.

[31] G.G. Kasparov. Equivariant KK-theory and the Novikov conjecture. Invent. math., 91:147201, 1988.

[32] R. Lauter, B. Monthubert, and V. Nistor. Pseudodifferential analysis on continuous family groupoids. Doc. Math., 5:625-655 (electronic), 2000.

[33] Jean-Marie Lescure. Elliptic symbols, elliptic operators and Poincaré duality on conical pseudomanifolds. Preprint. arXiv:math.OA/0609328.

[34] K. Mackenzie. Lie groupoids and Lie algebroids in differential geometry, volume 124 of London Mathematical Society Lecture Note. Cambridge university press, 1987.

[35] S. T. Melo, R. Nest, and E. Schrohe. $C^{*}$-structure and $K$-theory of Boutet de Monvel's algebra. J. Reine Angew. Math., 561:145-175, 2003.

[36] R.B. Melrose. The Atiyah-Patodi-Singer index theorem. Research Notes in Mathematics (Boston, Mass.). 4. Wellesley, MA: A. K. Peters, Ltd.. xiv, 377 p. , 1993.

[37] R.B. Melrose. Geometric scattering theory. Stanford Lectures. Cambridge University Press, Cambridge, 1995.

[38] Richard B. Melrose and Paolo Piazza. Analytic $K$-theory on manifolds with corners. Adv. Math., 92(1):1-26, 1992.

[39] B. Monthubert. Pseudodifferential calculus on manifolds with corners and groupoids. Proc. Amer. Math. Soc., 127(10):2871-2881, 1999.

[40] B. Monthubert and V. Nistor. A topological index theorem for manifolds with corners. Preprint '05.

[41] P. Muhly, J. Renault, and D. Williams. Equivalence and isomorphism for groupoid $C^{*}$ algebras. J. Operator Theory, 17(1):3-22, 1987.

[42] V. E. Nazaikinskii, A. Yu. Savin, and B. Yu. Sternin. On the homotopy classification of elliptic operators on stratified manifolds. Preprint, math.KT/0608332, 2006.

[43] V. E. Nazaikinskii, A. Yu. Savin, and B. Yu. Sternin. Pseudodifferential operators on stratified manifolds. Preprint, math.AP/0512025, 2006.

[44] V. E. Nazaŭkinskiı̌, A. Yu. Savin, B. Yu. Sternin, and B.-V. Shulze. On the index of elliptic operators on manifolds with edges. Mat. Sb., 196(9):23-58, 2005.

[45] V. Nistor. The index of operators on foliated bundles. J. Funct. Anal., 141(2):421-434, 1996.

[46] V. Nistor. Higher index theorems and the boundary map in cyclic cohomology. Doc. Math., 2:263-295 (electronic), 1997.

[47] V. Nistor. Groupoids and the integration of Lie algebroids. J. Math. Soc. Japan, 52:847-868, 2000 .

[48] V. Nistor, A. Weinstein, and P. Xu. Pseudodifferential operators on differential groupoids. Pacific J. of Math., 181(1):117-152, 1999.

[49] J. Renault. A groupoid approach to $C^{*}$-algebras, volume 793 of Lecture Notes in Math. Springer-Verlag, 1980.

[50] A. Savin. Elliptic operators on manifolds with singularities and $K$-homology. $K$-Theory, 34(1):71-98, 2005. 
[51] B.-W. Schulze. Pseudo-differential operators on manifolds with singularities, volume 24 of Studies in Mathematics and its Applications. North-Holland Publishing Co., Amsterdam, 1991.

[52] B.-W. Schulze. Pseudodifferential boundary value problems, conical singularities, and asymptotics, volume 4 of mathematical topics. Akademie Verlag, 1994.

[53] B.-W. Schulze, B. Sternin, and V. Shatalov. On the index of differential operators on manifolds with conical singularities. Ann. Global Anal. Geom., 16(2):141-172, 1998.

[54] A. Verona. Stratified mappings - structure and triangulability, volume 1102 of Lecture Notes in Mathematics. Springer-Verlag, Berlin, 1984.

[55] N. E. Wegge-Olsen. K-theory and $C^{*}$-algebras. Oxford Science Publications. The Clarendon Press Oxford University Press, New York, 1993. A friendly approach.

[56] H.E. Winkelnkemper. The graph of a foliation. Ann. Glob. Analysis and Geometry, 1 (3):51$75,1983$.

Laboratoire de Mathématique, Université Blaise Pascal, Campus universitaire des CÉzeaux, 63177 Aubière Cedex, France

E-mail address: debord@math.univ-bpclermont.fr

Laboratoire de Mathématique, Université Blaise Pascal, Campus universitaire des CÉzeaux, 63177 Aubière Cedex, France

E-mail address: lescure@math.univ-bpclermont.fr

Institutul de Matematică al Academiei Române and Department of Mathematics, Pennsylvania State University, University Park, PA 16802, USA

E-mail address: nistor@math.psu.edu 